\title{
ON THE REDUCTIVE BOREL-SERRE COMPACTIFICATION, III: MIXED HODGE STRUCTURES*
}

\author{
STEVEN ZUCKER ${ }^{\dagger}$
}

\begin{abstract}
We establish a procedure for constructing compatible mixed Hodge structures for the cohomology of various topological compactifications of locally symmetric varieties, notably ones that are not algebraic varieties. This is carried out in full for the case of the reductive Borel-Serre compactification, and conditionally for the excentric compactifications.
\end{abstract}

Introduction. In [BS], Borel and Serre gave a construction that compactified the arithmetic quotient $X$ of a symmetric space (also certain more general locally homogeneous spaces) to a manifold-with-corners $\bar{X}$. With its faces fibered by nilmanifolds, it was both an easy and a useful little construction to collapse each fiber to a point, producing what is now called the reductive Borel-Serre compactification $\bar{X}^{\text {red }}$ of $X$. For some of the uses of this space, see [Z1:§4], [GHM], [GT], [Z3].

The construction applies, in particular, to the Hermitian cases (i.e., locally symmetric varieties). Even there, the boundary faces are often of odd real dimension, which rules out the idea that $\bar{X}^{\text {red }}$, like $X$, be an algebraic variety over $\mathbb{C}$ or even a complex space.

Still, Mark Goresky once made a casual remark concerning $\bar{X}^{\text {red }}$ in the Hermitian case. I forget his exact words, but it was something like, "It thinks it's an algebraic variety." As a Hodge-theorist, I had the inevitable reaction: there should be a mixed Hodge structure on its cohomology. That was the impetus for this work. Here, we must remind the reader that the existence of a mixed Hodge structure on a vector space is not a significant statement; the selecting of one is.

In this article, after eight largely expository sections, we construct in $\S 9$ mixed Hodge structures for $H^{\bullet}\left(\bar{X}^{\text {red }}\right)$ when $X$ is Hermitian. We also sketch a construction of mixed Hodge structures for the cohomology of related compactifications of $X$. This includes first, in $\S 10, \bar{X}^{\mathrm{exc}}$ (the excentric Borel-Serre compactification), which is a quotient of $\bar{X}$ that maps to $\bar{X}^{\text {red }}$. As there are mappings (see [Z2], [GT], [Z4])

$$
\bar{X}^{\mathrm{exc}} \rightarrow \bar{X}^{\mathrm{red}} \rightarrow X^{*}
$$

where the last space is the projective algebraic Baily-Borel compactification [BB], we show that we get a morphism of mixed Hodge structure $H^{\bullet}\left(X^{*}\right) \rightarrow H^{\bullet}\left(\bar{X}^{\text {red }}\right)$, and conditionally also $H^{\bullet}\left(\bar{X}^{\text {red }}\right) \rightarrow H^{\bullet}\left(\bar{X}^{\text {exc }}\right)$. The condition here is completing the construction of the mixed Hodge structure on $H^{\bullet}\left(\bar{X}^{\text {exc }}\right)$; that seems to be obstructed by our inability to show that two mixed Hodge structures, on a cohomology group that enters, coincide (see (8.17)). In $\S 12$, we mention a possible resolution: generalizing the notion of mixed Hodge modules to spaces more general than complex varieties.

In a different direction are the smooth projective toroidal compactifications $X^{\text {tor }}$ of [AMRT], which lie in the realm of classical Hodge theory. However, these too have an excentric quotient $X^{\text {tor,exc }}$, which is generally not a complex space. On its

\footnotetext{
* Received June 27, 2004; accepted for publication November 30, 2004. jhu.edu).

$\dagger$ Department of Mathematics, Johns Hopkins University, Baltimore, MD 21218, USA (zucker@
} 
cohomology we also sketch the construction of a mixed Hodge structure in $\S 11$, such that

$$
H^{\bullet}\left(X^{*}\right) \rightarrow H^{\bullet}\left(X^{\text {tor, exc }}\right) \rightarrow H^{\bullet}\left(X^{\text {tor }}\right)
$$

would be morphisms of mixed Hodge structures (the same difficulty as for $\bar{X}^{\text {exc }}$ arises here). Since $\bar{X}^{\text {exc }}$ and $X^{\text {tor,exc }}$ are homotopy equivalent [Z4], hence have isomorphic cohomology, it is natural to expect that the mixed Hodge structures for $H^{\bullet}\left(\bar{X}^{\mathrm{exc}}\right)$ and $H^{\bullet}\left(X^{\mathrm{tor}, \mathrm{exc}}\right)$ will coincide.

The basic theme in our construction is to note that all of the above spaces have closed boundary strata that are of lower rank, but involve the same sort of compactification. Thereby, we can produce the mixed Hodge structure recursively.

The results of this article were treated provisionally in a talk given at the conference Algebraic Geometry 2000, held in July of that year in Azumino, Japan. ${ }^{1}$ The title of the talk was "The reductive Borel-Serre as an algebraic variety." I can picture how Borel would have reacted to that title in the absence of the backdrop, and I dedicate this work to his memory with respect and affection.

Acknowledgments. I am grateful to Les Saper for helpful correspondence. The referee is to be commended for careful and critical readings of earlier versions of the article, which led to great improvement in the exposition.

1. Topological preliminaries. Let $X$ be a locally compact Hausdorff space, and let $\widehat{X}$ be a compactification of $X$ (as a Hausdorff topological space.) Also, let $\widehat{j}: X \hookrightarrow \widehat{X}$ be the associated embedding, via which we identify $X$ with $\widehat{j}(X)$. We put $\partial \widehat{X}=\widehat{X}-X$, the boundary of $X$ in $\widehat{X}$, and let $\widehat{i}: \partial \widehat{X} \hookrightarrow \widehat{X}$ denote the inclusion.

If $\widehat{N}$ denotes an open neighborhood of $\partial \widehat{X}$ in $\widehat{X}$, we have the Mayer-Vietoris sequence for (say) rational cohomology:

$$
\cdots \rightarrow H^{\bullet}(\widehat{X}, \mathbb{Q}) \rightarrow H^{\bullet}(X, \mathbb{Q}) \oplus H^{\bullet}(\widehat{N}, \mathbb{Q}) \rightarrow H^{\bullet}(N, \mathbb{Q}) \rightarrow \ldots,
$$

where $N=\widehat{N} \cap X$. Under the hypothesis (rather mild in practice) that $\partial \widehat{X}$ is locally contractible, and has a fundamental system of neighborhoods that admit a deformation retraction onto $\partial \widehat{X},(1.1)$ yields the exact sequence:

$$
\cdots \rightarrow H^{\bullet}(\widehat{X}, \mathbb{Q}) \rightarrow H^{\bullet}(X, \mathbb{Q}) \oplus H^{\bullet}(\partial \widehat{X}, \mathbb{Q}) \rightarrow H_{\mathrm{dn}}^{\bullet}(\partial \widehat{X}, \mathbb{Q}) \rightarrow \ldots,
$$

where $H_{\mathrm{dn}}^{\bullet}$ denotes the so-called deleted neighborhood cohomology (the hypercohomology of the sheaf of nearby cycles). This can be expressed at the level of sheaves in terms of the standard functors: there is a canonical quasi-isomorphism

$$
\mathbb{Q}_{\widehat{X}} \approx\left\{R \widehat{j}_{*} \mathbb{Q}_{X} \oplus \widehat{i}^{*} \mathbb{Q}_{\widehat{X}} \rightarrow \widehat{i}^{*} R \widehat{j}_{*} \mathbb{Q}_{X}\right\} ;
$$

of course, we have by assumption that $\widehat{i}^{*} \mathbb{Q}_{\widehat{X}}=\mathbb{Q}_{\partial \widehat{X}}$. Here, $\mathbb{Q}_{\widehat{X}}$ and $\mathbb{Q}_{X}$ denote the constant sheaves on $\widehat{X}$ and $X$ with stalk $\mathbb{Q}$. We note that the hypercohomology of both $R \widehat{j}_{*} \mathbb{Q}_{X}$ and $\hat{i}^{*} R \widehat{j}_{*} \mathbb{Q}_{X}$ are independent of the compactification $\widehat{X}$, so one may work with them on any convenient choice of $\widehat{X}$.

\footnotetext{
${ }^{1}$ At the end of the talk, Morihiko Saito pointed out that the sort of notion of morphism of mixed Hodge complexes was the same one that A. Beilinson used in his fundamental article [Be]. In writing this article, I have discovered there is more than incidental relevance of that work.
} 
2. Hodge-theoretic preliminaries. We develop in some detail the fundamental Hodge theoretic notions.

In [De:III], Deligne introduced the notion of a mixed Hodge complex. Its definition was based on the means by which a mixed Hodge structure is typically determined on topologically-defined cohomology groups on algebraic varieties. It will be convenient to relax the notion of a morphism of mixed Hodge complexes in the way dictated by the principles of Hodge theory (cf. [Be]). We begin with a version that is easy to state, but is actually too loose:

(2.1) Definition. A (relaxed) mixed Hodge complex $C^{\bullet}$ is a triple of complexes $\left(C_{\mathbb{Z}}^{\bullet}, C_{W}^{\bullet}, C_{W, F}^{\bullet}\right)$, where

i) $C_{\mathbb{Z}}$ is a complex of free $\mathbb{Z}$-modules with finitely generated cohomology;

ii) $C_{W}^{\bullet}$ is a complex of $\mathbb{Q}$ vectorspaces with increasing filtration $W$.

iii) $C_{W, F}^{\bullet}$ is a complex of $\mathbb{C}$ vectorspaces with increasing filtration, also denoted $W$, and decreasing filtration $F$,

together with quasi-isomorphisms a) $C_{\mathbb{Z}}^{\bullet} \otimes_{\mathbb{Z}} \mathbb{Q} \rightarrow C_{W}^{\bullet}$, and b) $C_{W}^{\bullet} \otimes_{\mathbb{Q}} \mathbb{C} \rightarrow C_{W, F}^{\bullet}$ (respecting $W$ ), such that the filtrations induced by $W$ and $F$ define a mixed Hodge structure on

$$
H^{\bullet}\left(C_{W, F}^{\bullet}\right) \simeq H^{\bullet}\left(C_{\mathbb{Z}}^{\bullet}\right) \otimes_{\mathbb{Z}} \mathbb{C}
$$

In the mixed Hodge structure, $F^{p} H^{\bullet}\left(C^{\bullet}\right)$ is the image of $H^{\bullet}\left(F^{p} C^{\bullet}\right)$ in $H^{\bullet}\left(C^{\bullet}\right)$, the set of cohomology classes represented by an element of $F^{p} C^{\bullet}$; this defines the Hodge filtration. The weight filtration on $H^{i}\left(C^{\bullet}\right)$ is given with the usual shift:

$$
W_{k} H^{i}\left(C^{\bullet}\right)=i m\left\{H^{i}\left(D_{k} C^{\bullet}\right) \rightarrow H^{i}\left(C^{\bullet}\right)\right\},
$$

with $D$ as in $(2.2$,ii) below (in effect, one counts the degree $i$ as a contribution to the total weight). For the fundamental notion of a mixed Hodge structure, see [De:II, 2.3]; it expresses a certain type of relation between $F$ and $W$ on cohomology.

There are two essential ingredients that must be added to (2.1) in order to recover Deligne's definition:

(2.2) Definition. i) The differential $d$ in a complex $C^{\bullet}$ is said to be strictly compatible with a filtration $F$ if the inclusion

$$
F^{p} C^{\bullet}+\operatorname{ker} d \subseteq d^{-1}\left(F^{p} C^{\bullet}\right)
$$

is an equality.

ii) If $W$ is an increasing filtration of $C^{\bullet}$, the filtration $D=\operatorname{Dec}(W)$ [De:II, 1.3.3] is the convolved (amalgamated) filtration $W * \delta$, where $\delta$ is the filtration given by truncation from below (made increasing). Said another way, $D_{\ell} C^{i} \subseteq W_{\ell-i} C^{i}$, and $D_{\ell} C^{\bullet}$ is the largest subcomplex of $C^{\bullet}$ with that property.

We can now formulate the definition from [De:III, 8.1] as follows:

(2.3) Definition. A mixed Hodge complex $C^{\bullet}$ will be called strict if for each $k$, $\mathrm{G} r_{k}^{W} C^{\bullet}$, with the filtration induced by $F$, is a Hodge complex of weight $k$; i.e., if for each $j, F$ is strictly compatible with $d$ on $\mathrm{Gr}_{k}^{W} C^{\bullet}$, and the filtration induced by $F$ on $H^{j}\left(\mathrm{G} r_{k}^{W} C^{\bullet}\right)$ gives the latter a Hodge structure of weight $j+k$. Here, $\mathrm{G} r{ }_{k}^{W}$ denotes the successive quotient functor $W_{k} / W_{k-1}$. 
It follows from (2.3) that on the complex $C^{\bullet}$ itself, $d$ is strictly compatible with both $F$ and $\operatorname{Dec}(W)$ [De:III,(8.1.9)].

(2.4) Remarks. i) In the pure case, i.e., when $W$ is trivial, the notions of a Hodge filtration and a Hodge decomposition are equivalent.

ii) If one is willing to dispense with $\mathbb{Z}$-cohomology and work over $\mathbb{Q}$, one can simply suppress $C_{\mathbb{Z}}^{\bullet}$ in (2.1), and thus in (2.3). We shall do this throughout.

iii) Any mixed Hodge structure, viewed as a bifiltered complex with trivial differential, is a strict mixed Hodge complex. This observation will be used later.

We will not be so strict about morphisms:

(2.5) Definition. $A$ (relaxed) morphism of mixed Hodge complexes:

$$
\left(C_{\mathbb{Z}}^{\bullet}, C_{W}^{\bullet}, C_{W, F}^{\bullet}\right)=C^{\bullet} \rightarrow K^{\bullet}=\left(K_{\mathbb{Z}}^{\bullet}, K_{W}^{\bullet}, K_{W, F}^{\bullet}\right)
$$

is a triple of morphisms of complexes $\phi=\left(\phi_{\mathbb{Z}}, \phi_{W}, \phi_{W, F}\right)$, with $\phi_{\mathbb{Z}}: C_{\mathbb{Z}}^{\bullet} \rightarrow K_{\mathbb{Z}}^{\bullet}$, etc., such that $\phi_{W}$ and $\phi_{W, F}$ respect the corresponding filtrations, and also the quasiisomorphisms a) and b) in (2.1).

The main reason we use strict mixed Hodge complexes here is the following fact:

(2.6) Proposition. The collection of strict mixed Hodge complexes is closed under the following constructions:

i) The total complex of a double complex $\left(C^{\bullet}\right)^{\bullet}$, where the second differential gives morphisms of mixed Hodge complexes, as in (2.5), with filtrations: $F^{p}\left(C^{\bullet}\right)^{j}=\left(F^{p} C^{\bullet}\right)^{j}$ and $\widetilde{W}_{k}\left(C^{\bullet}\right)^{j}=\left(W_{k+j} C^{\bullet}\right)^{j}$.

ii) The complex of equivariant cochains $C^{\bullet}=C^{\bullet}\left(\Gamma ; K^{\bullet}\right)$, where $\Gamma$ is a group that acts on the strict mixed Hodge complex $K^{\bullet}$, respecting the filtrations. The filtrations of $C^{\bullet}$ are given by $F^{p} C^{j}=C^{j}\left(\Gamma ; F^{p} K^{\bullet}\right)$ and $\widetilde{W}_{k} C^{j}=C^{j}\left(\Gamma ; W_{k+j} K^{\bullet}\right)$.

Proof. We observe that i) is just [De:III, $(8.1 .15, \mathrm{i})]$, and the proof of ii) is similar; we give the latter. Let $S$ denote the increasing filtration by degree, i.e., the one for which $\operatorname{Gr}_{-j}^{S} C^{\bullet}=C^{j}[-j]$; here $[-j]$ says that degrees have been lowered by $j$. Then the weight filtration on $C^{\bullet}$ can be described as $\widetilde{W}=W * S$, so $\mathrm{Gr}^{\widetilde{W}} C^{\bullet}$ is naturally isomorphic to

$$
\bigoplus_{-j+\ell=k} \operatorname{Gr}_{-j}^{S} \operatorname{Gr}_{\ell}^{W} C^{\bullet}=\bigoplus_{\ell-j=k} \operatorname{Gr}_{\ell}^{W} C^{j}\left(\Gamma ; K^{\bullet}\right)[-j] .
$$

By assumption, $\operatorname{Gr}_{\ell}^{W} C^{j}$ is a Hodge complex of weight $\ell$. The effect of the shift is to lower the weight by $j$, so (2.6.1) is the sum of Hodge complexes of weight $k$, as required.

The following is standard:

(2.7) Proposition. Let $\phi: C^{\bullet} \rightarrow K^{\bullet}$ be a morphism of mixed Hodge complexes. Then the induced mapping on cohomology,

$$
H^{\bullet}(\phi): H^{\bullet}\left(C^{\bullet}\right) \rightarrow H^{\bullet}\left(K^{\bullet}\right)
$$

is a morphism of mixed Hodge structures. In particular, $H^{\bullet}(\phi)$ is strictly compatible with the filtrations induced by $D=\operatorname{Dec}(W)$ and $F$. 
(2.8) Corollary. If $\phi: C^{\bullet} \rightarrow K^{\bullet}$ is a morphism of mixed Hodge complexes that induces an isomorphism on cohomology, then $H^{\bullet}(\phi)$ is an isomorphism of mixed Hodge structures.

We are led to call a morphism $\phi$ as in (2.8) a quasi-isomorphism of mixed Hodge complexes (a quasi-isomorphism of certain bifiltered complexes), as distinguished from the stricter bifiltered quasi-isomorphism that requires $\operatorname{Gr}_{k}^{W} \mathrm{Gr}_{F}^{p} C^{\bullet} \rightarrow \operatorname{Gr}_{k}^{W} \mathrm{Gr}_{F}^{p} K^{\bullet}$ to be a quasi-isomorphism. We will work in the setting of strict mixed Hodge complexes and their relaxed morphisms.

Because (2.8) is true, one sees that bifiltered derived categories are too rigid a setting for working with the Hodge theory. The following examples illustrate this plainly.

(2.9) Examples. i) Let $K^{\bullet}$ be any complex, with increasing filtration $W$; forget about $F$. Let $K^{\bullet} \stackrel{I}{\rightarrow} K^{\bullet}$ be the identity mapping of $K^{\bullet}$. We adopt the convention that the underlying graded vector space of $C o n e(I)$ is

$$
C^{\bullet}=K^{\bullet} \oplus K^{\bullet}[-1],
$$

with differential

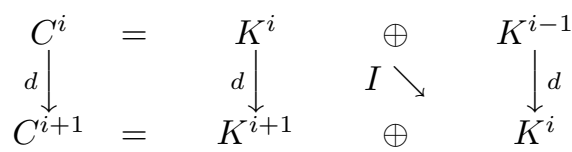

By definition of the filtered cone, $W_{k} C^{i}=W_{k} K^{i} \oplus W_{k+1} K^{i-1}$. The inclusion of the zero complex in $C^{\bullet}$ is a quasi-isomorphism respecting $W$. It is a filtered quasiisomorphism with respect to $W$ only when $K^{\bullet}$ is trivial, as

$$
\mathrm{Gr}_{k}^{W} C^{\bullet} \simeq \mathrm{Gr}_{k}^{W} K^{\bullet} \oplus \mathrm{Gr}_{k+1}^{W} K^{\bullet}[-1] .
$$

ii) We point out that if $C^{\bullet}$ and $K^{\bullet}$ are complexes with decreasing filtration $F$ given by truncation from below (filtration bête), then a morphism $C^{\bullet} \rightarrow K^{\bullet}$ is a filtered quasi-isomorphism with respect to $F$ if and only if $C^{\bullet}=K^{\bullet}$.

The following result holds in the context of general bifiltered complexes:

(2.10) Lemma. Let $C$ • be a bifiltered complex, with filtrations $W$ (increasing) and $F$ (decreasing). Then the following two statements are equivalent:

i) The inclusion $\left(F^{p} W_{k}\right) H^{\bullet}\left(C^{\bullet}\right) \rightarrow F^{p} H^{\bullet}\left(C^{\bullet}\right) \cap W_{k} H^{\bullet}\left(C^{\bullet}\right)$ is an isomorphism.

ii) For all $k$, p, there exists a bifiltered morphism $H^{\bullet}\left(C^{\bullet}\right) \rightarrow C^{\bullet}$ inducing the identity on cohomology.

Moreover, they are satisfied by strict mixed Hodge complexes.

Proof. The subject in (i) is $\left(F^{p} W_{k}\right) H^{\bullet}\left(C^{\bullet}\right)=i m\left\{H^{\bullet}\left(\left(F^{p} \cap W_{k}\right) C^{\bullet}\right) \rightarrow H^{\bullet}\left(C^{\bullet}\right)\right\}$. First, we note that if one of the filtrations, say $W$, is trivial, then (i) becomes a tautology, and (ii) is automatic (just take a cohomology basis that is filtered with respect to $F$ ). In general, (ii) gives the existence of bifiltered cohomology representatives, which clearly implies (i). One shows the converse recursively with respect to $W$. Start with a basis for $W_{\min } H^{\bullet}\left(C^{\bullet}\right)$, the lowest non-zero $W$-level, that is filtered with respect to $F$. From the definition, the mapping $W_{k} H^{\bullet}\left(C^{\bullet}\right) \rightarrow W_{k+1} H^{\bullet}\left(C^{\bullet}\right)$ is injective. Given a bifiltered basis of $W_{k} H^{\bullet}\left(C^{\bullet}\right)$, one uses (i) to extend it to a bifiltered basis of $W_{k+1} H^{\bullet}\left(C^{\bullet}\right)$, etc. 
Finally, for a strict mixed Hodge complex, the conditions are clearly satisfied by $\operatorname{Dec}(W)$ and $F$. Since $\operatorname{Dec}(W)$ and $W$ differ only by a shift of degree on cocycles, the conditions also hold for $W$ and $F$.

We note that $(2.10$,ii) is not functorial, and is taken without regard to group actions that might be present.

(2.11) Lemma (existence of good models). Let $C_{1}^{\bullet}$ and $C_{2}^{\bullet}$ be bifiltered complexes with filtrations $W$ and $F$, and suppose that $\left(H^{\bullet}\left(C_{1}^{\bullet}\right) ; W, F\right) \simeq\left(H^{\bullet}\left(C_{2}^{\bullet}\right) ; W, F\right)$. If the conditions of (2.10) hold for both $C_{1}^{\bullet}$ and $C_{2}^{\bullet}$, then there are bifiltered complexes $K^{\bullet}$ and $L^{\bullet}$ with sequences of quasi-isomorphisms of bifiltered complexes $K^{\bullet} \rightarrow C_{1}^{\bullet} \rightarrow L^{\bullet}$ and $K^{\bullet} \rightarrow C_{2}^{\bullet} \rightarrow L^{\bullet}$.

Proof. Clearly, we can take $K^{\bullet}=\left(H^{\bullet}\left(C_{1}^{\bullet}\right) ; W, F\right)$, as in (2.7). If we let

$$
L^{\bullet}=\operatorname{Cone}\left\{K^{\bullet} \rightarrow C_{1}^{\bullet} \oplus C_{2}^{\bullet}\right\}[1],
$$

the mixed cone on the diagonal embedding of $K^{\bullet}$, one sees rather easily that the required properties hold.

We call $K^{\bullet}$ and $L^{\bullet}$ bifiltered models of $C_{1}^{\bullet}$ and $C_{2}^{\bullet}$. The following variant of (2.11) comes rather easily:

(2.12) Proposition (models compatible with a set of morphisms). For $1 \leq j \leq$ $N+1$, let $C_{j}^{\bullet}$ be a bifiltered complex, such that their bifiltered cohomology $\left(H^{\bullet}\left(C_{j}^{\bullet}\right) ; W, F\right)$ are all isomorphic. Assume that the conditions of (2.10) hold for each $C_{j}^{\bullet}$. Suppose further that there are bifiltered morphisms $B_{j}^{\bullet} \rightarrow C_{j}^{\bullet}$. Then there is a bifiltered model $L^{\bullet}$ of the $C_{j}^{\bullet}$ 's such that all $B_{j}^{\bullet}$ map to $L^{\bullet}$.

Proof. Indeed, we may take

$$
L^{\bullet}=\text { Cone }\left\{\left(\bigoplus_{1 \leq j \leq N} K^{\bullet}\right) \rightarrow\left(\bigoplus_{1 \leq j \leq N+1} C_{j}^{\bullet}\right)\right\}[1],
$$

where the $j$-th factor of $K^{\bullet}$ maps to $C_{j}^{\bullet} \oplus C_{j+1}^{\bullet}$ as in (2.11). It is easy to see that $\left(H^{\bullet}\left(L^{\bullet}\right) ; W, F\right) \simeq\left(H^{\bullet}\left(C_{j}^{\bullet}\right) ; W, F\right)$ and each $B_{j}^{\bullet}$ maps to $L^{\bullet}$.

We conclude this section by introducing a notion that will be useful later $(\S 12)$ :

(2.13) Definition. A mixed Hodge complex $C^{\bullet}$ is said to be of complete type, when the weights in $H^{i}\left(C^{\bullet}\right)$ are $\leq i$; equivalently, it is of complete type when the mapping $H^{\bullet}\left(W_{0} C^{\bullet}\right) \rightarrow H^{\bullet}\left(C^{\bullet}\right)$ is surjective.

Clearly, a mixed Hodge complex with $W_{0} C^{\bullet}=C^{\bullet}$ is of complete type. The standard mixed Hodge complexes for complete algebraic varieties are, whence the name. We give a feature of the class of mixed Hodge complexes of complete type:

(2.14) Proposition. Let $A^{\bullet}$ and $B^{\bullet}$ be mixed Hodge complexes, and let $C^{\bullet}=$ Cone $\left\{A^{\bullet} \rightarrow B^{\bullet}\right\}$ be the mixed cone. Then,

i) if $A^{\bullet}$ and $B^{\bullet}$ are of complete type, then so is $C^{\bullet}$;

ii) if $C^{\bullet}$ and $B^{\bullet}$ are of complete type, then so is $A^{\bullet}$.

Proof. The assertions follow directly from the properties of the mixed cone. 
3. The construction (basic ingredient). We return now to the setting of $\S 1$. We further assume that $H^{\bullet}(X)$ and $H_{\mathrm{dn}}^{\bullet}(\partial \widehat{X})$ come with distinguished mixed Hodge structures, e.g., when $X$ is an algebraic variety over $\mathbb{C}$. There are two categories of triples of mixed Hodge complexes that enter, and we describe them next.

(3.1) Definitions. i) The category $\mathfrak{A}$ has as objects the set of triples $\mathcal{C}=$ $\left(C_{1}^{\bullet}, C_{2}^{\bullet}, C_{3}^{\bullet}\right)$, where $C_{2}^{\bullet}$ is a strict mixed Hodge complex with cohomology $H^{\bullet}(\partial \widehat{X})$, $C_{1}^{\bullet}$ is a strict mixed Hodge complex yielding the distinguished mixed Hodge structure on $H^{\bullet}(X)$, and $C_{3}^{\bullet}$ is a strict mixed Hodge complex yielding the distinguished mixed Hodge structure on $H_{\mathrm{dn}}^{\bullet}(\partial \widehat{X})$; furthermore, there are morphisms of mixed Hodge complexes $C_{2}^{\bullet} \rightarrow C_{3}^{\bullet}$ and $C_{1}^{\bullet} \rightarrow C_{3}^{\bullet}$ inducing the natural mappings $H^{\bullet}(\partial \widehat{X}) \rightarrow H_{\mathrm{dn}}^{\bullet}(\partial \widehat{X})$ and $H^{\bullet}(X) \rightarrow H_{\mathrm{dn}}^{\bullet}(\partial \widehat{X})$ respectively. A morphism $\mathcal{C} \rightarrow \mathcal{D}$ in $\mathfrak{A}$ is a morphism of triples such that the diagrams

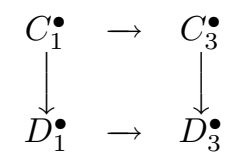

and

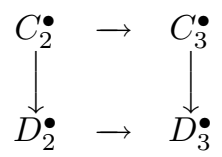

commute.

ii) The category $\mathfrak{B}$ has as objects the set of triples $\mathcal{K}=\left(C_{1}^{\bullet}, K_{2}^{\bullet}, C_{3}^{\bullet}\right)$, where $K_{2}^{\bullet}$ is a strict mixed Hodge complex with cohomology $H^{\bullet}(\widehat{X}), C_{1}^{\bullet}$ and $C_{3}^{\bullet}$ are as in (i) above; furthermore, there are morphisms of mixed Hodge complexes $K_{2}^{\bullet} \rightarrow C_{1}^{\bullet} \rightarrow C_{3}^{\bullet}$ inducing the canonical mappings $H^{\bullet}(\widehat{X}) \rightarrow H^{\bullet}(X) \rightarrow H_{\mathrm{dn}}^{\bullet}(\partial \widehat{X})$. A morphism $\mathcal{K} \rightarrow \mathcal{L}$ in $\mathfrak{B}$ is defined analogously to that in $\mathfrak{A}$.

Then we have:

(3.2) Proposition. The formula $K_{2}^{\bullet}=$ Cone $\left\{C_{1}^{\bullet} \oplus C_{2}^{\bullet} \rightarrow C_{3}^{\bullet}\right\}$ determines a functor from $\mathfrak{A}$ to $\mathfrak{B}$.

In effect, we can determine a mixed Hodge structure on $H^{\bullet}(\widehat{X})$ by doing the same for $H^{\bullet}(\partial \widehat{X})$ in a "sensible" manner.

4. Locally symmetric varieties and their toroidal compactifications. Let $X$ now be a locally symmetric variety. As an analytic space, it is of the form $\Gamma \backslash D$, where $D$ is a bounded symmetric domain and $\Gamma$ is an arithmetically-defined subgroup of the isometry group $G$. The latter means that there is an algebraic group $\mathcal{G}$ defined over $\mathbb{Q}$ with $\mathcal{G}(\mathbb{R}) \simeq G$ (up to compact factors), such that $\Gamma$ is commensurable with a(ny) determination of $\mathcal{G}(\mathbb{Z})$. The relevant constructions of compactifications of $X$ are all based on $\mathcal{G}$. It is also convenient to regard $\Gamma$ as a functor on the the set of algebraic subquotients of $\mathcal{G}$ :

$$
\Gamma\left(\mathcal{H}_{1} / \mathcal{H}_{2}\right)=\left(\Gamma \cap \mathcal{H}_{1}(\mathbb{Q})\right) /\left(\Gamma \cap \mathcal{H}_{2}(\mathbb{Q})\right) .
$$

When $\Gamma$ is neat [Bo: $\{17$ ], the above is torsion-free and depends only on the quotient $\mathcal{H}_{1} / \mathcal{H}_{2}$, not the pair $\left(\mathcal{H}_{1}, \mathcal{H}_{2}\right)$.

We shall be brief here, referring the reader to the literature for details. The toroidal compactifications $X^{\text {tor }}$ of $X$ are a class of compactifications defined by an elaborate construction with toroidal embeddings [AMRT]. That construction is determined from a collection $\Sigma$ of compatible simplicial cone complexes $\Sigma_{P}$ for the centers 
$U_{P}$ of the unipotent radicals $W_{P}$ of maximal $\mathbb{Q}$-parabolic subgroups $P$ of $G .^{2}$ For $\Gamma$ sufficiently small and $\Sigma$ suitably chosen, $X^{\text {tor }}$ will be a smooth projective variety. We will always assume that this holds.

The boundary $Z=\partial X^{\text {tor }}$ thus constructed is a divisor with normal crossings on $X^{\text {tor }}$. It is the union of closed subdivisors $Z(P)\left(Z_{P}\right.$ in [HZ1]) with normal crossings, where $P$ indexes the finite set of $\Gamma$-conjugacy classes of maximal $\mathbb{Q}$-parabolic subgroups of $G$ (or representatives thereof). These are the closed maximal boundary strata. The actual boundary $P$-stratum of $X^{\text {tor }}$, which arises from $U_{P}$, is denoted ${ }^{\circ} Z(P)$, for which we remove from $Z(P)$ all points lying in some $Z(Q)$ with $Q$ not conjugate to $P .^{3}$ (A hint of the construction of the $P$-stratum is present below.) Taking $P$ from a standard lattice of parabolic subgroups, we take the closed covering of $Z$ with elements ${ }^{4}$

$$
\check{Z}(P)=\bigsqcup\left\{Z\left({ }^{g} P\right): g \in \mathcal{G}(\mathbb{Q})\right\},
$$

and let $\mathcal{N}^{\text {tor }}$ be its nerve. For general reasons, $Z$ is homotopy equivalent to $\mathcal{N}^{\text {tor }}$.

For $R$ an arbitrary $\mathbb{Q}$-parabolic subgroup of $G$, i.e., not necessarily maximal, taken again modulo $\Gamma$-conjugacy, one defines $Z(R)$ and ${ }^{\circ} Z(R)$ in a parallel manner. Then $Z(R)$ enters into $\mathcal{N}^{\text {tor }}$ in the following way. Write

$$
R=\bigcap\{P: P \text { maximal, } P \supseteq R\} .
$$

Then $Z(R)$ is one connected component of $\bigcap Z(P)$ ( $P$ as above), and there are finitely many other components associated to certain $\mathcal{G}(\mathbb{Q})$-conjugates of $R$. As we allow $R$ to vary over all $\mathcal{G}(\mathbb{Q})$-conjugates, we get that $\mathcal{N}^{\text {tor }}$ consists of the sets

$$
\check{Z}(R)=\bigcap\{\check{Z}(P): P \text { maximal, } P \supseteq R\}=\bigsqcup\left\{Z\left({ }^{g} R\right): g \in \mathcal{G}(\mathbb{Q})\right\} .
$$

The association of $R$ to the "smallest" - with respect to the usual order relation " $\prec$ " that also relates boundary components (see [AMRT:p.240]) $-P$ in (4.2) is the relation of subordinacy, written as $P=\Pi(R)$. Said another, though related, way: for $P$ as above, there is a standard decomposition $P^{\text {red }}=P / W_{P} \simeq G_{\ell, P} \cdot G_{h, P}$ (see [AMRT: p.209]), and one selects from (4.2) the $P$ for which $G_{h, P}$ is smallest. Then ${ }^{\circ} Z(R)$ is constructed over $M_{P}$, a locally symmetric variety for the so-called boundary component $D_{P}$ of $D$ normalized by $P$ (homogeneous under $G_{h, P}$ ), from the mixed Shimura variety $M_{P}^{\prime}$ associated to $P$ (see, e.g., [HZ1:1.2.5]). Indeed, there is a diagram

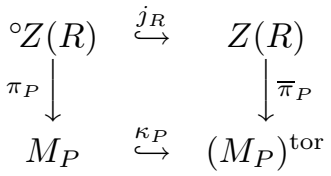

with the left-hand column given by the construction of $X^{\text {tor }}$. Every irreducible component of ${ }^{\circ} Z(R)$ maps onto $M_{P}$. The existence of a suitable toroidal compactification $\left(M_{P}\right)^{\text {tor }}$ of the lower-dimensional $M_{P}$ is provided by [P]; this falls in the setting of toroidal compactification of mixed Shimura varieties.

\footnotetext{
${ }^{2}$ Borel disliked the use of "parabolic" as a noun. I therefore use it only as an adjective.

${ }^{3}$ Designating as strata what we would call ${ }^{<} Z(P)$ here $\left({ }^{<} Z_{P}\right.$ in $[\mathrm{HZ1} 1)$ is adapted only to the mapping $X^{\text {tor }} \rightarrow X^{*}$.

${ }^{4}$ On the right-hand side, we have committed the abuse of notation that the union is disjoint in the sense that pairwise the indicated strata either coincide (by the $\Gamma$-action) or do not intersect.
} 
Each $Z(R)$ (equivalently, ${ }^{\circ} Z(R)$ ) has a combinatorial structure of its own, coming from $\Sigma$. We present a quick description. When $R=P$ (i.e., when $R$ is maximal), the irreducible components of $Z(P)$ are smooth divisors, denoted $Z_{\sigma}$, that are created by a one-dimensional cone (ray) $\sigma$ in the interior of $\Sigma_{P}$. Such $\sigma$ generate the subcomplex $\Sigma_{P}^{c}$ of $\Sigma_{P}$. In the compactification of $X$, this gets taken modulo the action of $\Gamma\left(G_{\ell, P}\right)$ : the torus $T_{P}=U_{P}(\mathbb{C}) / \Gamma\left(U_{P}\right)$ acts on $M_{P}^{\prime}$ and the $P$-stratum is produced from a $\Gamma\left(G_{\ell, P}\right)$-equivariant torus embedding $T_{P, \Sigma}$. For general $R$, put $Q=\Pi(R)$. Then the other maximal $P$ that occur in (4.2) have $U_{P} \subset U_{Q}$ (so $T_{P} \subset T_{Q}$ ), and $\Sigma_{P}$ is contained in the Satake boundary (see [HZ2:(2.1)]) of $\Sigma_{Q}$. By taking joins, one obtains subcomplexes $\Sigma_{R}^{c} \subset \Sigma_{R}$ of $\Sigma_{Q}$.

The following discussion takes place in $\Sigma_{Q}$. The set $Z_{\sigma}$ is defined for all $\sigma \in \Sigma_{Q}$, with intersections given by the rule

$$
Z_{\sigma} \cap Z_{\sigma^{\prime}}=Z_{\tau}
$$

where $\tau$ is the cone generated by $\sigma$ and $\sigma^{\prime}$ (when $\tau \in \Sigma_{Q}$; the intersection is empty otherwise). We can now assert:

(4.5) Proposition. The irreducible components of $Z(R)$ are of the form $Z_{\tau}=\bigcap\left\{Z_{\sigma(P)}: \sigma(P)\right.$ is an interior ray of $\Sigma_{P}$, and these generate a cone $\left.\tau \in \Sigma_{Q}\right\}$.

In the above, $P$ is as in (4.2). As one might expect, $\tau$ must be taken modulo $\Gamma\left(G_{\ell, R}\right)$, where $G_{\ell, R}=R \cap G_{\ell, P}$.

5. Deleted neighborhood cohomology for locally symmetric varieties: topological considerations. We return again to the setting of $\S 1$ when $X$ is a locally symmetric variety. It is convenient to start by taking $\widehat{X}=X^{\text {tor }}$, for there the complexes of sheaves $\mathrm{R} \widehat{j}_{*} \mathbb{Q}_{X}$ and $\widehat{i}^{*} \mathrm{R} \widehat{j}_{*} \mathbb{Q}_{X}$ underlie known cohomological mixed Hodge complexes (terminology of [De:III, 8.1.6]). We will drop the "hats" when working on the toroidal compactification.

Our purpose in this section is to give an account of the determination from [HZ2:§5]. Associated to the nerve $\mathcal{N}^{\text {tor }}$, we have a quasi-isomorphism

$$
i^{*} \mathrm{R} j_{*} \mathbb{Q}_{X} \approx \bigoplus_{R} \mathcal{T}_{R}[1-r(R)],
$$

where $\mathcal{T}_{R}=i_{R}^{*} \mathrm{R} j_{*} \mathbb{Q}_{X}, r(R)$ denotes the parabolic $\mathbb{Q}$-rank of $R$, and the right-hand side comes with a second differential given by restriction from $Z(R)$ to $Z\left(R^{\prime}\right)$ whenever $R^{\prime} \subset R$ with $r\left(R^{\prime}\right)=r(R)+1$. (When we write such direct sums, we always understand the presence of the restriction mappings.) For that, we note the simplification due to the nature of $Z(R)$ :

(5.2) Proposition. The sheaf $\mathcal{T}_{R}$, which is supported on $Z(R)$, is quasi-isomorphic to $\mathrm{R}\left(j_{R}\right)_{*}\left(j_{R}^{*} \mathcal{T}_{R}\right)$. Thus, $\mathcal{T}_{R}$ is determined by total direct image from its restriction to ${ }^{\circ} Z(R)$.

From (4.4) and (5.2) follows immediately:

(5.3) Corollary. Let $P=\Pi(R)$. Then the natural morphism

$$
\mathrm{R}\left(\bar{\pi}_{P}\right)_{*} \mathcal{T}_{R} \rightarrow \mathrm{R}\left(\kappa_{P}\right)_{*} \mathrm{R}\left(\pi_{P}\right)_{*}\left(j_{R}^{*} \mathcal{T}_{R}\right)
$$


is a quasi-isomorphism.

Let $i_{\tau}: Z_{\tau} \hookrightarrow X^{\text {tor }}$ be the inclusion. Taking the direct image by $\pi_{P}$, one obtains as $\tau$ varies the $\Gamma\left(G_{\ell, R}\right)$-equivariant collection of isomorphisms

$$
\mathrm{R} \pi_{*} j_{R}^{*} i_{\tau}^{*} \mathrm{R} j_{*} \mathbb{Q}_{X} \stackrel{\sim}{\sim} \mathbb{H}^{\bullet}\left(\mathfrak{w}_{P}, \mathbb{Q}\right),
$$

where $\mathbb{H}^{\bullet}\left(\mathfrak{w}_{P}, \mathbb{Q}\right)$ denotes the local system on $M_{P}$ associated to the representation of $G_{h, P}$ on the Lie algebra cohomology $H^{\bullet}\left(\mathfrak{w}_{P}, \mathbb{Q}\right) .^{5}$ Let $\Sigma_{R}^{o}$ be the collection of simplices that contain some $\tau$ (as in (4.5)) as a face. Combining (5.3) with (5.4), we obtain a quasi-isomorphism

$$
\begin{aligned}
\mathrm{R} \pi_{*} j_{R}^{*} i_{R}^{*} \mathrm{R} j_{*} \mathbb{Q}_{X} \approx \bigoplus_{\tau \in \Gamma\left(G_{\ell, R}\right) \backslash \Sigma_{R}^{o}} \mathbb{H}^{\bullet}\left(\mathfrak{w}_{P}, \mathbb{Q}\right) & \\
& \approx C^{\bullet}\left(\Gamma\left(G_{\ell, R}\right), \mathbb{H}^{\bullet}\left(\mathfrak{w}_{P}, \mathbb{Q}\right)\right),
\end{aligned}
$$

as $\Sigma_{R}^{o}$ is contractible (for it admits a simplicial collapse onto the contractible space $\Sigma_{P}^{o}$, where $P$ is the largest maximal parabolic subgroup entering in (4.2)). When we apply (5.3) to this, we get:

(5.6) Proposition. Let $P=\Pi(R)$. There is a canonical quasi-isomorphism:

$$
\mathcal{T}_{R}=i_{R}^{*} \mathrm{R} j_{*} \mathbb{Q}_{X} \approx \mathrm{R}\left(\kappa_{P}\right)_{*} C^{\bullet}\left(\Gamma\left(G_{\ell, R}\right), \mathbb{H}^{\bullet}\left(\mathfrak{w}_{P}, \mathbb{Q}\right)\right) .
$$

Because the relevant spectral sequence degenerates (see [HZ2:§5]), we obtain: ${ }^{6}$

(5.7) Corollary. On hypercohomology, the quasi-isomorphism in (5.6) induces an isomorphism

$$
H_{\mathrm{dn}}^{k}(Z(R), \mathbb{Q}) \simeq \bigoplus_{i+j=k} H_{\Gamma\left(G_{\ell, R}\right)}^{i}\left(M_{P}, \mathbb{H}^{j}\left(\mathfrak{w}_{P}, \mathbb{Q}\right)\right)
$$

(5.8) Remark. Using (5.6) in conjunction with (5.1), we also have:

$$
i^{*} \mathrm{R} j_{*} \mathbb{Q}_{X} \approx \bigoplus_{R} \mathrm{R}\left(\kappa_{\Pi(R)}\right)_{*} C^{\bullet}\left(\Gamma\left(G_{\ell, R}\right), \mathbb{H}^{\bullet}\left(\mathfrak{w}_{P}, \mathbb{Q}\right)\right)[1-r(R)] .
$$

6. The Borel-Serre boundary nerves. Given the direct relationship with the deleted neighborhood nerve for the boundary, it is convenient give a thorough treatment of the Borel-Serre spaces, $\bar{X}, \bar{X}^{\mathrm{exc}}$ and $\bar{X}^{\text {red }}$. These are successively quotient compactifications of $X$ :

$$
\bar{X} \rightarrow \bar{X}^{\mathrm{exc}} \rightarrow \bar{X}^{\mathrm{red}}
$$

The $R$-strata ( $R$ as in $\S 4$ ) of their respective boundaries are correspondingly related:

$$
e^{\prime}(R) \rightarrow e^{\prime}(R)^{\mathrm{exc}} \rightarrow e^{\prime}(R)^{\mathrm{red}} .
$$

\footnotetext{
${ }^{5}$ More suggestively, the underlying cochain complex, $C^{\bullet}\left(\mathfrak{w}_{P}, \mathbb{Q}\right)$, is a representation of $P$. It gives a variation of mixed Hodge structure on the mixed Shimura variety $M_{P}^{\prime}$, whose restriction to $M_{P}$ (as determined by the choice of Levi subgroup) is quasi-isomorphic to its cohomology, $\mathbb{H} \bullet\left(\mathfrak{w}_{P}, \mathbb{Q}\right)$. [Ko]

${ }^{6}$ See also (8.1) below for more on this.
} 
For the closed (compact) boundary strata one has likewise, and analogous to (6.1):

$$
\overline{e^{\prime}(R)} \rightarrow{\overline{e^{\prime}(R)}}^{\mathrm{exc}} \rightarrow{\overline{e^{\prime}(R)}}^{\mathrm{red}}
$$

these are, in fact, just Borel-Serre spaces for $e^{\prime}(R)$.

In the above and throughout, a "prime" on an " $e$ " always denotes an arithmetic quotient, as in $[\mathrm{BS}]$. That is, there are spaces with boundary attached to $D$,

$$
\bar{D} \rightarrow \bar{D}^{\text {exc }} \rightarrow \bar{D}^{\text {red }}
$$

with $\mathfrak{G}(\mathbb{Q})$-action, such that $(6.1)$ is the $\Gamma$-quotient of $(6.4)$; moreover, $(6.2)$ and (6.3) are the $\Gamma(R)$-quotients of the $R$-strata of (6.4), and these are given by

$$
e(R) \rightarrow e(R)^{\mathrm{exc}}=e(R) / U_{P}(\mathbb{R}) \rightarrow e(R)^{\mathrm{red}}=e(R) / W_{R}(\mathbb{R}),
$$

where $P=\Pi(R)($ as in $\S 4)$;

$$
\overline{e(R)} \rightarrow \overline{e(R)}^{\mathrm{exc}} \rightarrow \overline{e(R)}^{\mathrm{red}}
$$

which is $(6.4)$ for $e(R)$. We will start to suppress the " $(\mathbb{R})$ ", the indicator of real points of a group, and we hope this causes no confusion. The first mapping in (6.6) factors through $\overline{e(R)} / U_{P}$. For the purpose of determining (6.3), it is enough do the same for (6.6), as the arithmetic quotients can be taken afterwards.

Here is a quick description of the incidence relations in $\partial \bar{D}$ (see [BS:§5] for details). The inclusion of $e(R)$ in $\bar{X}$ is by means of limits of geodesic projection: $X \rightarrow X / A_{R}=$ $e(R)$, where $A_{R}$ denotes the split factor of the Langlands decomposition of $R$. The structure of $\overline{e(R)}$ is given by $^{7}$

$$
e(R) \simeq D_{R} \times W_{R}, \quad \text { and then } \overline{e(R)} \simeq \bar{D}_{R} \times W_{R}
$$

here, $D_{R}$ is a contractible space, homogeneous under $R^{\text {red }}=R / W_{R}$.

Replacing $X$ by $e(R)$, one has the analogous inclusion of $e\left(R^{\prime}\right)$ in $\overline{e(R)}$ whenever $R^{\prime} \subset R$, in a way that is compatible with the inclusion $e\left(R^{\prime}\right) \subset \bar{X}$. Taking the union as $R^{\prime}$ varies, this gives $\overline{e(R)} \subset \bar{X}$; analogously, $\overline{e\left(R^{\prime}\right)} \subset \overline{e(R)}$. When $R^{\prime}=R \cap Q$, where $Q$ is maximal and does not contain $R$, we have $e\left(R^{\prime}\right)=e(R) / A_{Q}$; because $Q$ is maximal parabolic, $A_{Q}$ is of dimension one.

For the excentric case, we need to bring in $P=\Pi(R)$, viewing $\overline{e(R)}$ as a subset of $\overline{e(P)}$. First, we assert:

(6.7) Proposition. We have for the spaces in (6.5), $e(R) \simeq D_{\ell, R} \times D_{P} \times W_{P}$, $e(R)^{\text {exc }} \simeq D_{\ell, R} \times D_{P} \times V_{P}$, and $e(R)^{\text {red }} \simeq D_{\ell, R} \times D_{P}$. Here, $D_{\ell, R}$ is homogeneous under $G_{\ell, R}$, and $V_{P}=W_{P} / U_{P}$. In particular, $e(R), e(R)^{\mathrm{exc}}$ and $e(R)^{\mathrm{red}}$ are all contractible.

This yields:

\footnotetext{
${ }^{7}$ The splitting off of the unipotent radical is something I had regarded as "well-known", whose truth I started to question. A proof of this appears in the appendix (6.A) to this Section.
} 
(6.8) Proposition. We have for the spaces in (6.6),

i) $\overline{e(R)} \simeq \overline{D_{\ell, R}} \times \bar{D}_{P} \times W_{P}$,

ii) $\overline{e(R)}^{\mathrm{exc}} \simeq{\overline{D_{\ell, R}}}^{\mathrm{e}}{\overline{D_{P}}}^{\mathrm{exc}} \times V_{P}$,

iii) $\overline{e(R)}^{\text {red }} \simeq{\overline{D_{\ell, R}}}^{\text {red }} \times{\overline{D_{P}}}^{\text {red }}$. $^{8}$

Of course, there are analogous assertions for $R^{\prime}$. We describe the inclusion $\overline{e\left(R^{\prime}\right)} \subset$ $\overline{e(R)}$ - the descent to the excentric and reductive quotients will be apparent - in terms of (6.8). With $R^{\prime}=R \cap Q$ as above, either $\Pi\left(R^{\prime}\right)=P$ or $\Pi\left(R^{\prime}\right)=Q$. The first case is very easy to handle: $\overline{e\left(R^{\prime}\right)} \subset \overline{e(R)}$ is induced by the inclusion $\overline{D_{\ell, R^{\prime}}} \subset \overline{D_{\ell, R}}$. The second (i.e., where $Q \prec P$ ) requires more explanation. Using (6.7) and writing $P(Q)=G_{h, P} \cap Q$ (notation as in [HZ3:1.5]), we obtain the decomposition

$$
e\left(R^{\prime}\right)=e(R) / A_{Q}=D_{\ell, R} \times\left(D_{P} / A_{Q}\right) \times W_{P}=D_{\ell, R} \times e(P(Q)) \times W_{P}
$$

The inclusion $e\left(R^{\prime}\right) \subset \overline{e(R)}$ is now seen to be induced by the identity mapping of $D_{\ell, R}$ and the inclusion of the Borel-Serre boundary face:

$$
D_{\ell, P(Q)} \times D_{Q} \times W_{P(Q)}=e(P(Q)) \subset \overline{D_{P}} .
$$

One can verify directly (it is also contained in (8.12) below) that

$$
W_{P(Q)} \times W_{P}=W_{Q(P)} \times W_{Q} .
$$

Continuing (6.9), we have

$$
e\left(R^{\prime}\right)=D_{\ell, R} \times\left(D_{\ell, P(Q)} \times D_{Q}\right) \times W_{P(Q)} \times W_{P} .
$$

We use (6.10) to rearrange the factors above to recover the analogue of (6.7) for $R^{\prime}$ :

$$
e\left(R^{\prime}\right) \simeq D_{\ell, R^{\prime}} \times D_{Q} \times W_{Q}, \quad \text { with } \quad D_{\ell, R^{\prime}} \simeq D_{\ell, R} \times D_{\ell, P(Q)} \times W_{Q(P)} .
$$

There is no problem passing to the closures, i.e., $\overline{e\left(R^{\prime}\right)} \subset \overline{e(R)}$, and then to the excentric and reductive quotients. We summarize the outcome:

(6.13) Proposition. Let $R^{\prime}=R \cap Q$, with $Q \prec P=\Pi(R)$. Then:

i) The inclusion of $\overline{e\left(R^{\prime}\right)}$ in $\overline{e(R)}$ is induced by the inclusion of $\overline{e(P(Q))}$ in $\bar{D}_{P}$.

ii) The inclusion of ${\overline{e\left(R^{\prime}\right)}}^{\mathrm{exc}}$ in $\overline{e(R)}^{\mathrm{exc}}$ is induced by the inclusion of $\overline{e(P(Q))}^{\mathrm{exc}}$ in $\bar{D}_{P}^{\text {exc }}$.

iii) The inclusion of ${\overline{e\left(R^{\prime}\right)}}^{\mathrm{red}}$ in $\overline{e(R)}^{\mathrm{red}}$ is induced by the inclusion of $\overline{e(P(Q))}^{\mathrm{red}}$ in $\bar{D}_{P}^{\text {red }}$.

Proof. These are straightforward. We note that for the case of the excentric quotients, we get from (6.10) that $V_{P(Q)} \times V_{P}=W_{Q(P)} \times V_{Q}$, and this equality is used to manipulate the unipotent radicals.

One can easily determine from (6.8) the boundary strata of the arithmetic quotients in (6.1), as well as the quotient mappings in (6.2). The group $\Gamma(R)$ acts on the

${ }^{8}$ Arithmetic quotients $X_{\ell, R}$ of $D_{\ell, R}$ appear in $\S 9$. Because $G_{\ell, R}$ is not a reductive group when $R \neq P,\left(\overline{X_{\ell, R}}\right)^{\text {red }}$ is then not a compactification of $X_{\ell, R}$, for one also collapses out the unipotent factor of $D_{\ell, R}$. For the same reason, mixed Shimura varieties can fail to have reductive or excentric Borel-Serre compactifications; this distinction comes up again in $\S 10$ and $\S 11$. 
tower (6.5) to produce (6.2) Because both $W_{R}$ and $U_{P}$ are normalized by $R$, the tower (6.2) is a tower of fibrations over $e^{\prime}(R)^{\text {red }}$, with fibers

$$
\Gamma\left(W_{R}\right) \backslash W_{R} \rightarrow \Gamma\left(V_{R}\right) \backslash V_{R} \rightarrow\{p t\},
$$

where $V_{R}=U_{P} \backslash W_{R}$. We claim that the same holds for the closed strata:

(6.15) Proposition. The tower (6.3) is a tower of fibrations over ${\overline{e^{\prime}(R)}}^{\text {red }}$, with fibers as in (6.14).

Proof. We split

$$
\overline{e(R)} \simeq \bar{D}_{R} \times W_{R} \rightarrow \bar{D}_{R}=\overline{e(R)}^{\mathrm{red}}
$$

in accordance with (6.A.1) below. This induces a splitting

$$
\overline{e(R)}^{\mathrm{exc}} \simeq \bar{D}_{R} \times V_{R},
$$

which lies between those of $\overline{e(R)}$ and $\overline{e(R)}^{\text {red }}$ in the above. By definition, the geodesic action of $R^{\text {red }}$ is the ordinary action of a Levi subgroup $L_{R}$ along a corresponding $L_{R^{-}}$ orbit. We choose $L_{R}$ to be defined over $\mathbb{Q}$, which is always possible (see [BT:(0.8)]). Then the action of $\Gamma(R)$ on $\overline{e(R)}$ decomposes as expected under the above splitting, likewise for $\overline{e(R)}$ exc . The argument proceeds as for (6.2).

At this point, we describe the boundary nerves for $\bar{X}, \bar{X}^{\text {exc }}$ and $\bar{X}^{\text {red }}$. This goes basically the same way as the toroidal nerve in $\S 4$. Let $P$ be a maximal $\mathbb{Q}$-parabolic subgroup taken from a fixed standard lattice. We have

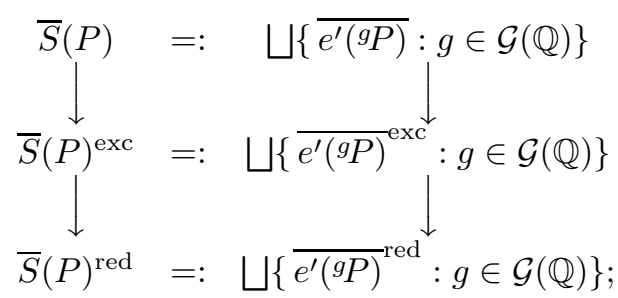

on the right-hand side, we commit the same abuse of notation as in (4.1). Then $\{\bar{S}(P): P$ is maximal $\}$ is a closed covering of $\partial \bar{X}$, whose nerve we denote by $\overline{\mathcal{N}}$; we do likewise to obtain nerves $\overline{\mathcal{N}}^{\text {exc }}$ for $\partial \bar{X}^{\text {exc }}$ and $\overline{\mathcal{N}}^{\text {red }}$ for $\partial \bar{X}^{\text {red }}$. For any $R$ in the lattice, we have

$$
\bar{S}(R)=\bigcap\{\bar{S}(P): P \text { maximal, } P \supseteq R\}=\bigsqcup\left\{\overline{e^{\prime}\left({ }^{g} R\right)}: g \in \mathcal{G}(\mathbb{Q})\right\},
$$

The analogous holds for $\bar{S}(R)^{\mathrm{exc}}$ and $\bar{S}(R)^{\mathrm{red}}$.

From (6.15) we obtain, for instance, that $\overline{e^{\prime}(R)}$ is an Eilenberg-Maclane space $K(\Gamma(R), 1)$, and its cohomology is given by the right-hand side of (5.7). The latter is no coincidence; as $\bar{X}$ is a manifold-with-corners, one can identify

$$
H^{\bullet}\left(\overline{e^{\prime}(R)}, \mathbb{Q}\right) \stackrel{\sim}{\longrightarrow} H_{\mathrm{d} n}^{\bullet}\left(\overline{e^{\prime}(R)}, \mathbb{Q}\right),
$$

so the previous assertion concerning $\left.H^{\bullet} \overline{e^{\prime}(R)}, \mathbb{Q}\right)$ is about an isomorphism of deleted neighborhood cohomology. While it is true that $H_{\mathrm{dn}}^{\bullet}$ for the total boundary is automatically independent of the compactification (as in $\S 1$ ), it is non-trivial that this remains true at the level of nerves for $\bar{X}$ and $X^{\text {tor }}$, for there is seldom a morphism of 
compactifications from one to the other. Nonetheless, the correspondence of nerves is proved in $[\mathrm{HZ} 2: \S 2]$. We thereby obtain:

(6.18) Proposition. There are canonical mappings

$$
\left.\left.H^{\bullet}{\overline{e^{\prime}(R)}}^{\mathrm{red}}, \mathbb{Q}\right) \rightarrow H^{\bullet}{\overline{e^{\prime}(R)}}^{\mathrm{exc}}, \mathbb{Q}\right) \rightarrow H^{\bullet}\left(\overline{e^{\prime}(R)}, \mathbb{Q}\right) \simeq H_{\mathrm{dn}}^{\bullet}(Z(R), \mathbb{Q}) .
$$

(The determination of the rightmost member in (6.18) is given by (5.7).)

The space $\mathcal{A}_{P}$ (see [HZ1:(1.2.5)]) over $M_{P}$ that enters in the toroidal construction is an arithmetic quotient of $D_{P} \times V_{P}$. As such, it admits a Borel-Serre compactification $\overline{\mathcal{A}_{P}}$, which maps to $\overline{M_{P}}$. The excentric quotient $\overline{\mathcal{A}_{P}}$ exc can be taken, and that is an arithmetic quotient of $\overline{D_{P}}{ }^{\text {exc }} \times V_{P}$. (On the other hand, there is no compactification " $\overline{\mathcal{A}}_{P}$ red" unless $V_{P}$ is trivial; recall $(6.8)$.) We observe that $(6.8$, ii) yields the description of $\overline{e^{\prime}(R)}$ exc as the free $\Gamma\left(G_{\ell, R}\right)$-quotient of $\overline{D_{\ell, R}} \times{\overline{\mathcal{A}_{P}}}^{\text {exc }}$, for the action of $\Gamma\left(G_{\ell, R}\right)$ on $\overline{D_{\ell, R}}$ is already free. On the other hand, the action of $\Gamma\left(G_{\ell, R}\right)$ on $\mathcal{A}_{P}$ is not nice. This is the realm of the Borel construction and equivariant cohomology, a quick account of which can be found in [HZ1:2.9]. At issue is replacing $\mathcal{A}_{P}$ by a homotopy-equivalent space $\mathcal{Q}$ on which the action of $\Gamma\left(G_{\ell, R}\right)$ is free. We denote by $\left\langle\Gamma\left(G_{\ell, R}\right), \mathcal{A}_{P}\right\rangle$ the common homotopy type of the spaces $\Gamma\left(G_{\ell, R}\right) \backslash \mathcal{Q}$, any of which we call a model for the Borel construction.

Because $\overline{D_{\ell, R}}$, being a manifold-with-corners whose interior $D_{\ell, R}$ is contractible, is itself contractible, we obtain:

(6.19) Proposition. The excentric boundary stratum $e^{\prime}(R)^{\mathrm{exc}}$ is a model for the Borel construction $\left\langle\Gamma\left(G_{\ell, R}\right), \mathcal{A}_{P}\right\rangle$ for the action of $\Gamma\left(G_{\ell, R}\right)$ on $\mathcal{A}_{P}$. Likewise, the closed excentric boundary stratum $\overline{e^{\prime}(R)}{ }^{\mathrm{exc}}$ is a model for $\left\langle\Gamma\left(G_{\ell, R}\right),{\overline{\mathcal{A}_{P}}}^{\mathrm{exc}}\right\rangle$.

(6.20) Corollary. There are canonical isomorphisms

$$
H^{\bullet}\left(e^{\prime}(R)^{\mathrm{exc}}\right) \simeq H_{\Gamma\left(G_{\ell, R}\right)}^{\bullet}\left(\mathcal{A}_{P}\right) \text {, and } H^{\bullet}\left({\overline{e^{\prime}(R)}}^{\mathrm{exc}}\right) \simeq H_{\Gamma\left(G_{\ell, R}\right)}^{\bullet}\left({\overline{\mathcal{A}_{P}}}^{\mathrm{exc}}\right) .
$$

(6.21) Remark. Likewise, $(6.8, \mathrm{i})$ gives the more elementary fact that $\overline{e^{\prime}(R)}$ is a model for $\left\langle\Gamma\left(G_{\ell, R}\right), \overline{M_{P}^{\prime}}\right\rangle$, where $P=\Pi(R)$. Furthermore, the inclusion $e^{\prime}\left(R^{\prime}\right) \subset \overline{e^{\prime}(R)}$ is given by

$$
\left\langle\Gamma\left(G_{\ell, R}\right),\left\langle\Gamma\left(G_{\ell, P(Q)} \cdot W_{Q(P)}\right), M_{Q}^{\prime}\right\rangle\right\rangle \rightarrow\left\langle\Gamma\left(G_{\ell, R}\right), \overline{M_{P}^{\prime}}\right\rangle,
$$

if $\Pi\left(R^{\prime}\right)=Q \prec P$.

(6.A) Appendix. For our construction, the following fact is essential. The proof we give is basically due to L. Saper.

(6.A.1) Proposition. Let $D$ be a homogeneous space for the real points of an algebraic group $\mathcal{G}$ that satisfies the conditions of [BS:§2]. Let $W$ be the unipotent radical of $\mathcal{G}$, and $D^{\prime}=W(\mathbb{R}) \backslash D=: W \backslash D$. Then:

i) The parabolic subgroups $\widehat{Q}$ of $W \backslash \mathcal{G}$ are in one-to-one correspondence with the parabolic subgroups $Q$ of $\mathcal{G}$, by the formula $\widehat{Q}=W \backslash Q$.

ii) The geodesic action of $A_{Q}$ on $D$ projects onto the geodesic action of $A_{\widehat{Q}}$ on $D^{\prime}$. 
iii) $D$ splits as $D^{\prime} \times W$ with respect to the geodesic actions, such that the action on $W$ is trivial.

iv) The above induces a splitting $\bar{D} \simeq \bar{D}^{\prime} \times W$.

Proof. While the argument that follows holds in general, and we even need to use that, we will treat for simplicity of notation only the case where $D$ is a symmetric space, viewed as a homogeneous space for $P$, where $P$ is a parabolic subgroup of $\mathcal{G}$. As before, write $P^{\text {red }}$ for $P / W_{P}$ and note that $D^{\prime}$ is homogeneous under $P^{\text {red }}$.

First, i) and ii) are in Borel-Serre [BS: 5.2]. Then, according to [S: $\S 1]$, the geodesic action of $A_{P}$ on $D$ extends to a geodesic action of $P^{\text {red }}$, one that commutes with the usual action of $W_{P}$. Taking quotients by these actions, we obtain a canonical $\left(P^{\text {red }} \times W_{P}\right)$-equivariant diffeomorphism

$$
D \stackrel{\sim}{\longrightarrow}\left(W_{P} \backslash D\right) \times\left(P^{\text {red }} \backslash D\right)
$$

that separates the actions of $P^{\text {red }}$ and $W_{P}$. One identifies $W_{P} \backslash D$ with $D^{\prime}$, and $\mathcal{W}=$ $P^{\text {red }} \backslash D$ is a principal homogeneous space for $W_{P}$. Thus $D \simeq D^{\prime} \times \mathcal{W}$. To get the same for the Borel-Serre construction, we again use [BS: 5.2] and note that the geodesic action is trivial on $\mathcal{W}$. Finally, to identify $\mathcal{W}$ and $W_{P}$, we need a section $D^{\prime} \rightarrow D$ for (6.A.2). Any point of $\mathcal{W}$ corresponds to an orbit of the geodesic action of $P^{\text {red }}$ on $D$, and gives a section. This yields both iii) and iv).

7. The excentric toroidal boundary nerve. The strata of the boundary of $X^{\text {tor }}$ are, of course, the spaces ${ }^{\circ} Z(R)$, where $R$ is $\mathbb{Q}$-parabolic, and the closed strata are the $Z(R)$ (see $\S 4$ ). The excentric toroidal compactifications $X^{\text {tor,exc }}$ are defined as the quotients of the toroidal compactifications $X^{\text {tor }}$ for which the boundary strata are the spaces ${ }^{\circ} Z(R)^{\text {exc }}={ }^{\circ} Z(R) / U_{P}$, with $P=\Pi(R)$. Their closures $Z(R)^{\text {exc }}$ comprise the boundary nerve $\mathcal{N}^{\text {tor,exc }}$ for $X^{\text {tor,exc }}$ (cf. $\mathcal{N}^{\text {tor }}$; recall (4.1) and (4.3)), so we need to understand their structure.

The space $\mathcal{A}_{P}$, introduced after (6.18), is actually a (connected) mixed Shimura variety (cf. $\S 4)$, so admits toroidal compactifications $\left(\mathcal{A}_{P}\right)^{\text {tor }}[\mathrm{P}]$ and then their excentric quotients $\left(\mathcal{A}_{P}\right)^{\text {tor,exc }}$; these are related in the same way as $X^{\text {tor }}$ and $X^{\text {tor,exc }}$. There is a $\Gamma\left(G_{\ell, R}\right)$-equivariant structure diagram (refining the excentric version of (4.4)):

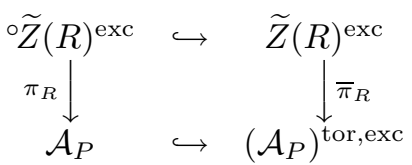

Then ${ }^{\circ} Z(R)^{\text {exc }} \simeq \Gamma\left(G_{\ell, R}\right) \backslash{ }^{\circ} \widetilde{Z}(R)^{\text {exc }}$, and $Z(R)^{\text {exc }}$ is defined analogously. We have the toroidal analogue of $(6.19)$ :

(7.2) Proposition. Let $\Pi(R)=P$. Then ${ }^{\circ} Z(R)^{\text {exc }}$ is a model for $\left\langle\Gamma\left(G_{\ell, R}\right), \mathcal{A}_{P}\right\rangle$, and $Z(R)^{\text {exc }}$ is a model for $\left\langle\Gamma\left(G_{\ell, R}\right),\left(\mathcal{A}_{P}\right)^{\text {tor,exc }}\right\rangle$.

Proof. We need to show that:

i) ${ }^{\circ} \widetilde{Z}(R)^{\text {exc }}$ and $\widetilde{Z}(R)^{\text {exc }}$ are homotopy equivalent to $\mathcal{A}_{P}$ and $\left(\mathcal{A}_{P}\right)^{\text {tor,exc }}$ resp.;

ii) the action of $\Gamma\left(G_{\ell, R}\right)$ on $\widetilde{Z}(R)^{\text {exc }}$ is free.

We start with the second assertion. To justify (ii), we must go a little deeper into the toroidal construction that underlies $\S 4$. This begins with writing $D$ as a Siegel domain 
relative to $P$, and then taking the quotient by (the real points of) $U_{P}$ :

$$
D / U_{P} \simeq\left(D_{P} \times V_{P}\right) \times C_{P},
$$

where $C_{P}$ is a cone homogeneous under $G_{\ell, P}$ (see [AMRT: p. 250]). Though the quotients by the $U_{P}$ 's are actually taken only at the corresponding toroidal boundary faces in making $X^{\text {tor,exc }}$ from $X^{\text {tor }}$, we find it more convenient to indicate the quotient in the interior as well, intending to undo it later.

The excentric toroidal construction (prior to the arithmetic quotients) gives

$$
\begin{array}{ccc}
\circ \widetilde{Z}(R)^{\operatorname{exc}} & \hookrightarrow & \left(D_{P} \times V_{P}\right) \times \widehat{\Sigma}_{P} \\
& \searrow & \downarrow \\
& D_{P} \times V_{P}
\end{array}
$$

where $\widehat{\Sigma}_{P}$ denotes $\Sigma_{P}-\{0\}$ modulo dilations, etc., and ${ }^{\circ} \widetilde{Z}(R)^{\operatorname{exc}}$ is contained in $\widetilde{Z}(P)^{\text {exc }}$. Let $Q$ be the smallest maximal parabolic subgroup in some standard lattice containing $P$. The closure of ${ }^{\circ} \widetilde{Z}(R)^{\text {exc }}$ is fibered over $D_{Q} \times V_{Q}$ with fiber equal to a $\Gamma\left(G_{\ell, R}\right)$-equivariant regular neighborhood of $\widehat{\Sigma}_{R}^{c}$ in $\widehat{\Sigma}_{Q}$. Then $\widehat{\Sigma}_{R}^{c}$ admits a free $\Gamma\left(G_{\ell, R}\right)$-action, as does its regular neighborhood. This holds for all such $Q$, so (ii) is true. ${ }^{9}$

Next, the fibers of $\pi_{P}$ are real torus embeddings (see [HZ1:2.1]) for $T_{P}$, thus contractible, so ${ }^{\circ} \widetilde{Z}(R)^{\text {exc }} \sim \mathcal{A}_{P}$. We must then look at the boundary fibers over $\left(\mathcal{A}_{P}\right)^{\text {tor,exc }}$. For all $Q \prec P$, the torus $T_{P}$ splits off as a factor of $T_{Q}$. Since the two factors commute, it follows that for all $Q$, the fibers over the $G_{h, Q}$-stratum of $\left(\mathcal{A}_{P}\right)^{\text {tor,exc }}$ are also real torus embeddings for $T_{P}$. Thus, $\widetilde{Z}(R)^{\text {exc }} \sim\left(\mathcal{A}_{P}\right)^{\text {tor,exc }}$.

8. The deleted neighborhood cohomology: Hodge-theoretic considerations. We return to the setting of $\S 5$. We want to summarize the known Hodgetheoretic facts that comprise the backdrop of this article. The main one is:

(8.1) THEOREM [HZ2:§5]. The isomorphism in (5.7) is an isomorphism of mixed Hodge structures over $\mathbb{Q}$ :

$$
H_{\mathrm{dn}}^{k}(Z(R), \mathbb{Q}) \simeq \bigoplus_{i+j=k} H_{\Gamma\left(G_{\ell, R}\right)}^{i}\left(M_{P}, \mathbb{H}^{j}\left(\mathfrak{w}_{P}, \mathbb{Q}\right)\right) .
$$

In particular, this gives a Hodge-theoretic direct sum decomposition of $H_{\mathrm{dn}}^{\bullet}(Z(R), \mathbb{Q})$.

Also, as was mentioned in (6.18), (8.1.1) is naturally isomorphic to $H^{k}\left(e^{\prime}(R), \mathbb{Q}\right)$. Through this isomorphism, one gets mixed Hodge structures for the Borel-Serre boundary faces. (These enter in $\S 9$ below.)

We clarify the content of (8.1) by describing the mixed Hodge structures on the two sides. One way to do that is via the mixed Hodge modules of Saito [Sa1]. In the present setting, Saito's construction proves more tractable than in general [BW], where (8.1.1) is shown to be a consequence of a determination in a derived category of mixed Hodge modules.

On the other hand, it is possible to specify the mixed Hodge structure of $H_{\mathrm{dn}}^{k}(Z(R))$ entirely by "classical" mixed Hodge theory [NA] (i.e., without the Hodge

\footnotetext{
${ }^{9}$ Note that the features are like those of $\overline{e\left(G_{\ell, R}\right)} \subset \overline{D_{\ell, Q}}$ (combine $\S 6$ above with [Z4:(3.3)]).
} 
modules), for we are considering (at least initially) cohomology with trivial coefficients, and we do so here. This is most easily treated when $R$ is maximal, or even better, for the full boundary divisor $Z$, and we describe only the latter case now. It begins with "turning" (1.3) to yield the quasi-isomorphism:

$$
i^{*} \mathrm{R} j_{*} \mathbb{Q}_{X} \approx \text { Cone }\left\{\mathbb{Q}_{X^{\text {tor }}} \rightarrow \mathrm{R} j_{*} \mathbb{Q}_{X} \oplus \mathbb{Q}_{Z}\right\}[1] .
$$

The mixed Hodge theory for each term of this mapping cone is well-known. One can take $C_{W, F}^{\bullet}=\mathrm{R}^{\boldsymbol{\bullet}} \mathfrak{C}_{W, F}^{\bullet}$ using the cohomological Hodge complexes $\mathfrak{C}_{W, F}^{\bullet}$, given respectively by $\Omega_{X^{\text {tor }}}^{\bullet}$ for $\mathbb{Q}_{X^{\text {tor }}}, \Omega_{X^{\text {tor }}}^{\bullet}(\log Z)$ for $\mathrm{R} j_{*} \mathbb{Q}_{X}$, and $\Omega_{Z}^{\bullet}$ from the nerve of its irreducible components:

$$
\Omega_{Z}^{\bullet} \approx \bigoplus_{R} \bigoplus\left\{\Omega_{Z_{\sigma}}^{\bullet}: \sigma \in\left(\Gamma\left(G_{\ell, R}\right) \backslash \Sigma_{R}^{o}\right)\right\}[1-r(R)]
$$

for $\mathbb{Q}_{Z}$. These are equipped with their usual weight and Hodge filtrations $W$ : trivial, by number of poles, and $S$ (as in the proof of (2.5)), resp.; and $F$ by filtration bête (from $(2.9, \mathrm{ii})$ ) in all three instances. The mixed cone construction (see [E:0, 2.6]) then tells us to use

$$
\mathfrak{C}_{W, F}^{\bullet}=\text { Cone }\left\{\Omega_{X^{\text {tor }}}^{\bullet} \rightarrow \Omega_{X^{\text {tor }}}^{\bullet}(\log Z) \oplus \Omega_{Z}^{\bullet}\right\}
$$

in the cohomological mixed Hodge complex for $i^{*} \mathrm{R} j_{*} \mathbb{Q}_{X}$, with filtrations induced by the convolved filtration $W * S$ (here $S$ is as in the proof of (2.5)) and $F$.

The mixed Hodge structure on the right-hand side in (8.1.1) can be seen to come from $(2.5, \mathrm{ii})$. It was introduced in a somewhat ad hoc way in [HZ2:(5.6)], via the right-hand side of (5.5) above. We give a sketch: through the action of $A_{P} G_{h, P}$, the local system $\mathbb{H} \bullet\left(\mathfrak{w}_{P}, \mathbb{Q}\right)$ also underlies a locally homogeneous variation of mixed Hodge structure on $M_{P}$, on which $G_{\ell, P}$, a fortiori $\Gamma\left(G_{\ell, R}\right)$ whenever $R$ is subordinate to $P$, acts. This variation of mixed Hodge structure splits as a direct sum of pure variations of varying weight. Then

$$
\bigoplus_{\sigma \in \Gamma\left(G_{\ell, R}\right) \backslash \Sigma_{R}^{o}} \mathbb{H}^{\bullet}\left(\mathfrak{w}_{P}, \mathbb{Q}\right)
$$

underlies a cosimplicial object of that sort. It is necessary to do the following little exercise concerning the forgetting of the filtration $S$ :

(8.6) Proposition. For the constant bifiltered sheaf $\mathbf{K}^{\bullet}=\mathbb{H} \bullet\left(\mathfrak{w}_{P}, \mathbb{Q}\right)$ on the finite-dimensional simplicial complex (from (8.5)),

$$
\bigoplus_{\sigma} \mathbf{K}^{\bullet} \rightarrow\left(\bigoplus_{\sigma} \mathbb{Q}\right) \otimes \mathbf{K}^{\bullet}
$$

where $\bigoplus_{\sigma} \mathbb{Q}$ on the right-hand side is viewed as a complex with trivial filtrations, induces the same variation of mixed Hodge structure.

Proof. We need only observe that the mapping above is compatible with $W$ and $F$, and the filtrations induce variations of mixed Hodge structure on both sides. We then invoke (2.8) for the stalks.

Assembling (5.5), (8.6) and standard Hodge theory, we get that a mixed Hodge complex $B^{\bullet}(R)$ for $i_{R}^{*} \mathrm{R} j_{*} \mathbb{Q}_{X}$ is obtained from

$$
\mathfrak{B}_{\mathbb{Q}}^{\bullet}(R)=C^{\bullet}\left(\Gamma\left(G_{\ell, R}\right), \mathrm{R}\left(\kappa_{P}\right)_{*} \mathbb{H}^{\bullet}\left(\mathfrak{w}_{P}, \mathbb{Q}\right)\right)
$$


on $\left(M_{P}\right)^{\text {tor }}$, with $B_{\mathbb{Q}}^{\bullet}(R)=\mathrm{R} \Gamma\left(\left(M_{P}\right)^{\text {tor }}, \mathfrak{B}_{\mathbb{Q}}^{\bullet}(R)\right)$, and

$$
B_{W, F}^{\bullet}(R)=C^{\bullet}\left(\Gamma\left(G_{\ell, R}\right), \operatorname{R} \Gamma\left(\left(M_{P}\right)^{\text {tor }}, \Omega_{\left(M_{P}\right)^{\text {tor }}}\left(\log Y_{P}\right) \otimes \mathcal{H}^{\bullet}\left(\mathfrak{w}_{P}, \mathbb{C}\right)^{\text {can }}\right) ;\right.
$$

here, $P=\Pi(R)$ again, and $Y_{P}$ denotes the total boundary divisor of the toroidal compactification $\left(M_{P}\right)^{\text {tor }}$ of $M_{P}$ (the choice of which is immaterial), and "can" indicates the canonical bundle-extension to $\left(M_{P}\right)^{\text {tor }}$ (see, e.g., [Z4:(4.2)]). The Hodge and weight filtrations in (8.8) come from the de Rham complex and coefficients, as given in [Sa2]. One deduces a mixed Hodge complex $B^{\bullet}$ for $i^{*} \mathrm{R} j_{*} \mathbb{Q}_{X}$ that is decomposed according to the toroidal boundary nerve $\mathcal{N}^{\text {tor }}$.

To complete the picture, we make explicit the restriction mappings $B^{\bullet}(R) \rightarrow$ $B^{\bullet}\left(R^{\prime}\right)$ for $r\left(R^{\prime}\right)=r(R)+1$, for they enter into the total differential of $B^{\bullet}$. We give a treatment vaguely parallel to that in $\S 6$. The easy case is when $\Pi\left(R^{\prime}\right)=\Pi(R)$; there, the restriction is induced by the inclusion

$$
\Gamma\left(G_{\ell, R^{\prime}}\right) \hookrightarrow \Gamma\left(G_{\ell, R}\right)
$$

in the obvious way. When $\Pi\left(R^{\prime}\right)=Q \prec P=\Pi(R)$, the description is similar, though a little more complicated. We present it at the level of $B_{\mathbb{Q}}^{\bullet}$, but the Hodge theory virtually "takes care of itself" ([HZ2], [BW]).

We first note that $M_{Q}$ is a boundary component of $M_{P}$, corresponding to the maximal parabolic subgroup $P(Q)$ of $G_{h, P}$. Let $\bar{\pi}_{P(Q)}:\left(M_{P}\right)^{\text {tor }} \rightarrow\left(M_{Q}\right)^{\text {tor }}$ be the associated projection (we will not go into the details here of the extent to which $\bar{\pi}_{P(Q)}$ is defined; see, e.g., [HZ1:1.2.5], but it is analogous to $\bar{\pi}_{P}$, which one uses to arrive at (8.7)). Taking the total direct image of (8.7) under $\bar{\pi}_{P(Q)}$ yields the complex on $M_{Q}$ :

$$
\widehat{\mathfrak{B}}_{\mathbb{Q}}^{\bullet}(R)=\mathrm{R}\left(\kappa_{Q}\right)_{*} C^{\bullet}\left(\Gamma\left(G_{\ell, R}\right), H^{\bullet}\left(\mathfrak{w}_{P(Q)}, \mathbb{H} \bullet\left(\mathfrak{w}_{P}, \mathbb{Q}\right)\right)\right) .
$$

This will be shown to admit a canonical mapping to

$$
\mathfrak{B}_{\mathbb{Q}}^{\bullet}\left(R^{\prime}\right)=C^{\bullet}\left(\Gamma\left(G_{\ell, R^{\prime}}\right), \mathrm{R}\left(\kappa_{Q}\right)_{*} \mathbb{H}^{\bullet}\left(\mathfrak{w}_{P}, \mathbb{Q}\right)\right),
$$

which is (8.7) for $R^{\prime}$ instead of $R$.

In order to reconcile (8.10) and (8.11), we first need some equations in algebraic groups:

(8.12) Proposition. For $Q \prec P$, let $P(Q)=Q \cap G_{h, P}, Q(P)=P \cap G_{\ell, Q}$ and $R^{\prime}=R \cap Q$. Then there are decompositions:

i) $W_{P} \cdot W_{P(Q)}=W_{P \cap Q}=W_{Q} \cdot W_{Q(P)}$,

ii) $G_{\ell, R^{\prime}}=G_{\ell, R} \cdot G_{\ell, P(Q)} \cdot W_{Q(P)}$,

iii) $G_{\ell, R^{\prime}} \cdot W_{Q}=G_{\ell, R} \cdot G_{\ell, P(Q)} \cdot W_{P \cap Q}$.

Proof. We verify only the first equality in (i), which suffices because of symmetry, and then (ii). (From these, (iii) follows.) We want to show first that

$$
W_{P \cap Q}=W_{P} \cdot\left(W_{Q} \cap G_{h, P}\right) .
$$

At the level of Lie algebras, this is saying that $\mathfrak{w}_{P \cap Q}=\mathfrak{w}_{P} \oplus \mathfrak{w}_{P(Q)}$; a positive root involving at least one of $\beta_{P}$ and $\beta_{Q}$ is either a root involving $\beta_{P}$ or a root involving $\beta_{Q}$ but not $\beta_{P}$. This gives (i). 
As for (ii), it is correct on Levi factors, so it suffices to compare the unipotent radicals of both sides, i.e., show:

$$
W_{G_{\ell, R^{\prime}}}=W_{G_{\ell, R}} \cdot W_{Q(P)},
$$

or equivalently

$$
\left(W_{R^{\prime}} \cap G_{\ell, Q}\right)=\left(W_{R} \cap G_{\ell, Q}\right)=\left(W_{R} \cap G_{\ell, P}\right) \cdot\left(W_{P} \cap G_{\ell, Q}\right)
$$

But this holds for basically the same reason that (8.12.1) does.

The other ingredient is the abundance of cohomology isomorphisms. In our setting, the cohomology of a free arithmetic quotient is canonically isomorphic to the group cohomology of the arithmetic group; moreover, in the case of a unipotent algebraic group, the cohomology is also isomorphic (by Nomizu's theorem [No]) to the corresponding Lie algebra cohomology group. Thus we have, for instance, quasiisomorphisms

$$
\mathrm{R} \Gamma\left(M_{P}, \mathbb{Q}\right) \approx C^{\bullet}\left(\Gamma\left(G_{h, P}\right), \mathbb{Q}\right) \quad \text { and } \quad C^{\bullet}\left(\mathfrak{w}_{P}, \mathbb{Q}\right) \approx C^{\bullet}\left(\Gamma\left(W_{P}\right), \mathbb{Q}\right) .
$$

Thus, from (8.12), we see that (8.10) is quasi-isomorphic to

$$
\mathrm{R}\left(\kappa_{Q}\right)_{*} C^{\bullet}\left(\Gamma\left(G_{\ell, R}\right), \mathbb{H}^{\bullet}\left(\mathfrak{w}_{P \cap Q}, \mathbb{Q}\right)\right) .
$$

Consider the diagram

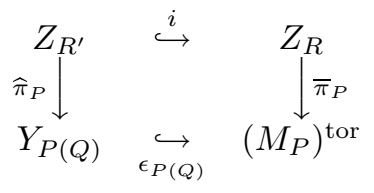

(cf. (4.4)). The quasi-isomorphism

$$
\mathrm{R}\left(\widehat{\pi}_{P}\right)_{*} i * \mathbb{S} \approx \epsilon_{P(Q)}^{*} \mathrm{R}\left(\bar{\pi}_{P}\right)_{*} \mathbb{S}
$$

yields, when $\mathbb{S}=i_{R}^{*} \mathrm{R} j_{*} \mathbb{Q}$ :

$$
\mathrm{R}\left(\widehat{\pi}_{P}\right)_{*} i_{R^{\prime}}^{*} \mathrm{R} j_{*} \mathbb{Q} \approx C^{\bullet}\left(\Gamma\left(G_{\ell, R}\right), \epsilon_{P(Q)}^{*} \mathrm{R}\left(\kappa_{P}\right)_{*} \mathbb{H}^{\bullet}\left(\mathfrak{w}_{P}, \mathbb{Q}\right)\right) .
$$

This has basically the same format as (8.7), viz., deleted neighborhood cohomology on $\left(M_{P}\right)^{\text {tor }}$ with coefficients in a local system on $M_{P}$. Moreover, there is an obvious mapping from (8.7) to (8.15). We therefore apply $\mathrm{R}\left(\bar{\pi}_{P(Q)}\right)_{*}$ to $(8.15)$, which yields

$$
\begin{aligned}
& \mathrm{R}\left(\bar{\pi}_{Q}\right)_{*} i_{R^{\prime}}^{*} \mathrm{R} j_{*} \mathbb{Q} \\
& \approx \mathrm{R}\left(\kappa_{Q}\right)_{*} C^{\bullet}\left(\Gamma\left(G_{\ell, R}\right), C^{\bullet}\left(\Gamma\left(G_{\ell, P(Q)}\right), \mathbb{H}^{\bullet}\left(\mathfrak{w}_{P(Q)}, H^{\bullet}\left(\mathfrak{w}_{P}, \mathbb{Q}\right)\right)\right)\right. \\
& \quad \approx \mathrm{R}\left(\kappa_{Q}\right)_{*} C^{\bullet}\left(\Gamma\left(G_{\ell, R}\right), C^{\bullet}\left(\Gamma\left(G_{\ell, P(Q)}\right), \mathbb{H}^{\bullet}\left(\mathfrak{w}_{P \cap Q}, \mathbb{Q}\right)\right)\right) \\
& \quad \approx C^{\bullet}\left(\Gamma\left(G_{\ell, R^{\prime}}\right), \mathrm{R}\left(\kappa_{Q}\right)_{*} \mathbb{H}^{\bullet}\left(\mathfrak{w}_{Q}, \mathbb{Q}\right)\right),
\end{aligned}
$$

recovering (8.11). Moreover, from (8.10) and the above, the restriction mapping $H_{\mathrm{dn}}^{\bullet}(Z(R), \mathbb{Q}) \rightarrow H_{\mathrm{dn}}^{\bullet}\left(Z\left(R^{\prime}\right), \mathbb{Q}\right)$ is seen to be induced by the inclusion $\{1\} \rightarrow \Gamma\left(G_{\ell, P(Q)}\right)$.

Because there is a mixed Hodge module on $X^{\text {tor }}$ underlying the above calculations, the two different-looking mixed Hodge complexes for $H^{\bullet}\left(Z_{R^{\prime}}\right)$, which follow respectively from (8.15) and (8.16), actually determine the same mixed Hodge structure. This leads us to make the following: 
(8.17) Definition. In any situation where there is a diagram analogous to (8.14), and (8.15) and (8.16) determine mixed Hodge structures on cohomology, we say that the change of base from $M_{P}$ to $M_{Q}$ (or between $P$ and $Q$ ) is Hodge theoretical when the two mixed Hodge structures coincide.

9. Mixed Hodge structures in the reductive Borel-Serre case. To standardize the notation, we use the set-up of $\S 3$. We start with one specification of mixed Hodge complexes $A^{\bullet}$ and $C^{\bullet}$ for which:

$$
\begin{aligned}
& A_{\mathbb{Q}}^{\bullet}:=C_{1, \mathbb{Q}}^{\bullet} \approx \mathrm{R} \Gamma\left(X^{\text {tor }}, \mathrm{R} j_{*} \mathbb{Q}_{X}\right), \\
& \widehat{C}_{\mathbb{Q}}^{\bullet}:=\widehat{C}_{3, \mathbb{Q}}^{\bullet} \approx \mathrm{R} \Gamma\left(X^{\text {tor }}, i^{*} \mathrm{R} j_{*} \mathbb{Q}_{X}\right), \quad \text { with decomposed version } \\
& C_{\mathbb{Q}}^{\bullet}:=C_{3, \mathbb{Q}}^{\bullet}=\bigoplus_{R} C^{\bullet}(R)_{\mathbb{Q}}, \quad \text { where }
\end{aligned}
$$

$C^{\bullet}(R)_{\mathbb{Q}} \approx \mathrm{R} \Gamma\left(X^{\text {tor }}, i_{R}^{*} \mathrm{R} j_{*} \mathbb{Q}_{X}\right) \approx C^{\bullet}\left(\Gamma\left(G_{\ell, R}\right), \mathrm{R} \Gamma\left(\left(M_{P}\right)^{\text {tor }}, \mathrm{R}\left(\kappa_{P}\right)_{*} \mathbb{H}^{\bullet}\left(\mathfrak{w}_{P}, \mathbb{Q}\right)\right)\right)$. Note that we have morphisms $A_{\mathbb{Q}}^{\bullet} \longrightarrow \widehat{C}_{\mathbb{Q}}^{\bullet} \stackrel{\approx}{\approx} C_{\mathbb{Q}}^{\bullet}$. Of course, we have to provide the rest of the items in (2.1) to complete (9.1) to mixed Hodge complexes.

To produce an element of category $\mathfrak{A}(3.1, \mathrm{i})$, we need mixed Hodge complexes $B^{\bullet}$ (denoted $C_{2}^{\bullet}$ in $\S 3$ ) that map to $C^{\bullet}$. They will usually be decomposed as $B^{\bullet}=$ $\bigoplus_{R} B^{\bullet}(R)$, with $B^{\bullet} \rightarrow C^{\bullet}$ given as the direct sum of morphisms $B^{\bullet}(R) \rightarrow C^{\bullet}(R)$ commuting with restriction mappings. Ultimately we will specify that the cohomology of $B^{\bullet}$ is $H^{\bullet}(\partial \widehat{X}, \mathbb{Q})$, where $\widehat{X}$ is some given compactification of $X$, for we want to produce mixed Hodge complexes for $H^{\bullet}(\widehat{X}, \mathbb{Q})$.

(9.2) Remark. It may help to consider first the familiar "extreme" examples:

i) Setting $B^{\bullet}(R)=0$ for all $R$ gives a complex whose cohomology is $H_{c}^{\bullet}(X, \mathbb{Q})$;

ii) $B^{\bullet}(R)=C^{\bullet}(R)$ gives a complex whose cohomology is $H^{\bullet}(\bar{X}, \mathbb{Q}) \stackrel{\sim}{\longrightarrow} H^{\bullet}(X, \mathbb{Q})$.

The construction of the mixed Hodge complexes is recursive, i.e., by induction on the $\mathbb{Q}$-rank $r$ (a non-negative integer), for the boundary of the compactifications that we are considering is always composed of pieces that are the same sort of compactifications of spaces of lower $\mathbb{Q}$-rank. The induction starts with $r=0$, where there is nothing to do ( $X$ is compact).

We will be making extensive use of strictness, i.e. (2.7), throughout. Because equivariant cochains enter in our other two constructions, the present case is the only one where we can appeal to (2.10) and thereby work solely at the level of mixed Hodge structures. Here and in the sequel, we write the subscript " $h$ " on a cohomology group to signify that it carries an existing mixed Hodge structure; cohomology without the subscript " $h$ " imparts a trivial Hodge theoretical contribution. Following the principle from [HZ2] that $G_{h, P}$ is Hodge-theoretically "active", while $G_{\ell, P}$ is Hodgetheoretically inert (cf. §8), we now present:

(9.3) Theorem (mixed Hodge complexes for the reductive Borel-Serre). Let $X$ be a locally symmetric variety. Take

$$
\begin{aligned}
A^{\bullet} & =H^{\bullet}(\bar{X})_{h} \simeq H^{\bullet}(X)_{h}, \\
C^{\bullet}(R) & =H^{\bullet}\left(\Gamma\left(G_{\ell, R}\right), H^{\bullet}\left(\overline{M_{P}^{\prime}}\right)_{h}\right), \\
B^{\bullet}(R) & =B^{\bullet}(R)^{\mathrm{red}}=H^{\bullet}\left(\left(\overline{X_{\ell, R}}\right)^{\mathrm{red}}\right) \otimes H^{\bullet}\left({\overline{M_{P}}}^{\mathrm{red}}\right)_{h},
\end{aligned}
$$


where $H^{\bullet}\left(\left(\overline{X_{\ell, R}}\right)^{\text {red }}\right)$ has trivial filtrations and $H^{\bullet}\left(\left(\overline{M_{P}}\right)^{\text {red }}\right)_{h}$ is the mixed Hodge structure for $H^{\bullet}\left(\left(\overline{M_{P}}\right)^{\text {red }}\right)$ already constructed.

Let $A^{\bullet} \rightarrow C^{\bullet}$ be the given by the restriction mapping $H^{\bullet}(X) \rightarrow H_{\mathrm{dn}}^{\bullet}(Z(R)$ ) (see (6.14)) for $R$ maximal; the mapping $B^{\bullet}(R) \rightarrow C^{\bullet}(R)$ is defined to be the composition

$$
\begin{aligned}
H^{\bullet}\left(\left(\overline{X_{\ell, R}}\right)^{\text {red }}\right) \otimes H^{\bullet}\left({\overline{M_{P}}}^{\mathrm{red}}\right) & \rightarrow H^{\bullet}\left(\left(\overline{X_{\ell, R}}\right) \otimes H^{\bullet}\left(\overline{M_{P}}\right) \simeq\right. \\
& H^{\bullet}\left(\Gamma\left(G_{\ell, R}\right), H^{\bullet}\left(\overline{M_{P}}\right)_{h}\right) \rightarrow H^{\bullet}\left(\Gamma\left(G_{\ell, R}\right), H^{\bullet}\left(\overline{M_{P}^{\prime}}\right)_{h}\right) .
\end{aligned}
$$

Then the above data is in the category $\mathfrak{A}$ and determines a mixed Hodge complex $C^{\bullet}\left(\bar{X}^{\text {red }}\right)_{h}$ for $H^{\bullet}\left(\bar{X}^{\text {red }}\right)$, such that the restrictions $H^{\bullet}\left(\bar{X}^{\text {red }}\right) \rightarrow H^{\bullet}\left(B^{\bullet}(R)^{\text {red }}\right)$ to a closed boundary stratum and $H^{\bullet}\left(\bar{X}^{\text {red }}\right) \rightarrow H^{\bullet}(\bar{X}) \simeq H^{\bullet}(X)$ are morphisms of mixed Hodge structure. Here, $H^{\bullet}(X)$ is understood to have Deligne's mixed Hodge structure for the algebraic variety $X$.

Proof. From (6.8), we see that $B^{\bullet}(R) \simeq H^{\bullet}\left({\overline{e^{\prime}(R)}}^{\text {red }}\right)$. Also, $C^{\bullet}(R)$, as given above, is consistent with (8.1.1) and (9.1); moreover it equals

$$
H^{\bullet}\left(X_{\ell, R} \times M_{P}, \mathbb{H}^{\bullet}\left(\mathfrak{w}_{P}\right)\right) \simeq H^{\bullet}\left(e^{\prime}(R)\right) .
$$

To see that $B^{\bullet} \rightarrow C^{\bullet}$ is a morphism of mixed Hodge complexes, we must demonstrate recursively that the following three statements hold:

i) For all $R, B^{\bullet}(R)^{\text {red }} \rightarrow C^{\bullet}(R)$ is a morphism of strict mixed Hodge complexes. This is the composition (9.3.1). By induction, $H^{\bullet}\left({\overline{M_{P}}}^{\text {red }}\right) \rightarrow H^{\bullet}\left(\overline{M_{P}}\right)$ is a morphism of mixed Hodge structures, which implies that $B^{\bullet}(R)^{\text {red }} \rightarrow C^{\bullet}(R)$ is.

ii) The canonical diagram

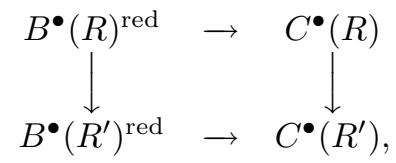

where $R^{\prime}=R \cap Q$ with $Q$ maximal $(Q \nsupseteq R)$, is a commutative diagram of mixed Hodge complexes. The diagram is explicitly

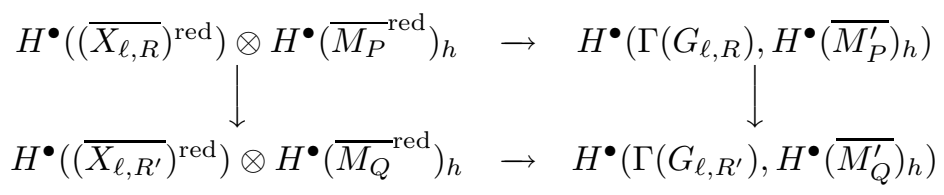

In the case where $\Pi\left(R^{\prime}\right)=P$, the assertion is evident, so we consider the other possibility, where $\Pi\left(R^{\prime}\right)=Q \prec P$. Using $(8.12$,ii), we rewrite the underlying cohomology groups in (9.3.2) relative to $P$ :

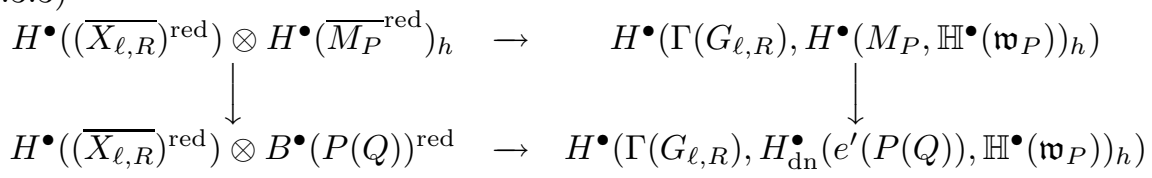

Then we see that (9.3.3) too is a diagram of mixed Hodge structures, and we need to know that the mixed Hodge structures, and in particular the one in the lower right corner, are the same as in (9.3.2). This is true by [Sa1]. 
iii) Let $R^{\prime \prime}=R \cap Q \cap Q^{\prime}\left(Q \nsupseteq R, Q^{\prime} \nsupseteq R\right)$. For both $\bar{X}^{\mathrm{red}}$ and $\bar{X}$, the diagram of complexes

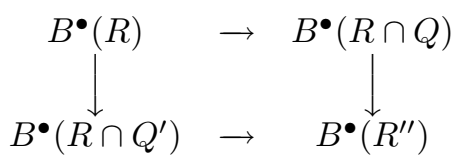

commutes. This is to ensure that $B^{\bullet}$ actually has a square-zero differential, and it is not a Hodge theoretical issue. Since we are working at the level of cohomology, this is not an issue here.

Finally, the restriction mappings to $H^{\bullet}(X)$ and to the cohomology of a closed boundary stratum are morphisms of mixed Hodge structure because they are induced by the natural projection of $\operatorname{Cone}\left\{A^{\bullet} \oplus B^{\bullet} \rightarrow C^{\bullet}\right\}$ onto $A^{\bullet} \oplus B^{\bullet}$.

(9.3.4) Remark. Using the $B^{\bullet}(R)^{\text {red }}$ 's is tantamount to giving a mixed Hodge complex for the nerve $\overline{\mathcal{N}}^{\text {red }}$ (from (6.16)).

We henceforth understand by the mixed Hodge structure on $H^{\bullet}\left(\bar{X}^{\text {red }}\right)$ to be the one determined by (9.3). We state for emphasis that no ordering of boundary strata needs to be specified beyond the instruction that the mixed Hodge structures are constructed "from the boundary inward", i.e., in the order of increasing $\mathbb{Q}$-rank.

Because $\bar{X}^{\text {red }}$ maps onto the Baily-Borel compactification $X^{*}$ of $X$, and $X^{*}$ is an algebraic variety, it is natural to ask whether the mixed Hodge structure constructed in (9.3) is compatible with Deligne's mixed Hodge structure on the cohomology of $X^{*}$. The answer is affirmative (as one should demand):

(9.4) Proposition. The mapping $H^{\bullet}\left(X^{*}\right) \rightarrow H^{\bullet}\left(\bar{X}^{\mathrm{red}}\right)$ is a morphism of mixed Hodge structures.

Proof. As always, this is verified recursively. The algebraic variety $X^{*}$ has an existing mixed Hodge structure on its cohomology. For comparing it to that of $\bar{X}^{\text {red }}$, we want a construction of a mixed Hodge complex for $H^{\bullet}\left(X^{*}\right)_{h}$ that follows the lines of $\S 9$, one for which we can appeal to $(2.8)$.

We note that the compactification $X^{*}$ differs substantially from the other ones we are considering in this article, in particular $\bar{X}^{\text {red }}$, in that the combinatorial nature of its boundary is different. This can be explained prior to taking the $\Gamma$-quotient, and we can work within a standard lattice of parabolic subgroups. For $\bar{D}^{\text {red }}$, the maximal closed boundary faces are of the form $\overline{e(P)}^{\text {red }}$ with $P$ maximal (in the sense of parabolic subgroups). The general face is of the form $\overline{e(R)}^{\text {red }}$, the intersection of $k$ of the maximal faces, corresponding to writing $R$ as the intersection of $k$ maximal parabolic subgroups, as in (4.2). On the other hand, the role of the non-maximal parabolic subgroups in $D^{*}$ is somewhat obscure, and we prefer to leave it that way. For $P$ maximal parabolic, the corresponding closed stratum is $\left(D_{P}\right)^{*}$. However, these are nested: (in the irreducible case) the maximal $P$ are linearly ordered under the relation $\prec(\S 4)$, whereby $P^{\prime} \prec P$ if and only if $\left(D_{P^{\prime}}\right)^{*} \subset\left(D_{P}\right)^{*}$. It may seem redundant to talk about intersections of these, but it is wise to do so.

We view the boundary strata of $\bar{X}^{\text {red }}$ in clusters, as in $\overline{\mathcal{N}}^{\text {red }}$. Recall that for each maximal $P$ in the lattice, $\bar{S}(P)^{\text {red }}$ denotes the disjoint union of all closed boundary strata associated to the conjugates of $P$. Then, let $S(P)^{*}$ be the image of $\bar{S}(P)^{\text {red }}$ in 
$\partial X^{*}$, a union (but not a disjoint union) of the $\left(D_{g_{P}}\right)^{*}$ s. If $R$ is parabolic, with $R=$ $\bigcap P$ again, then $\bar{S}(R)^{\mathrm{red}}=\bigcap \bar{S}(P)^{\mathrm{red}}$. Its image in $\partial X^{*}$ is $S(R)^{*}=: \bigcap S(P)^{*}$, which is an algebraic variety. The redundancy noted above gives us that $S(R)^{*}$ coincides with $S(\Pi(R))^{*}$.

Let $\mathcal{N}^{*}$ denote the corresponding nerve for $\partial X^{*}$. The mappings

$$
\bar{S}(R)^{\mathrm{red}} \rightarrow S(R)^{*}
$$

are compatible with inclusions of parabolic subgroups, so they induce a mapping of boundary nerves $\overline{\mathcal{N}}^{\text {red }} \rightarrow \mathcal{N}^{*}$. We get a decomposed mixed Hodge complex

$$
B^{\bullet}\left(X^{*}\right) \simeq \bigoplus_{R} B^{\bullet}(R)^{*}
$$

with cohomology $H^{\bullet}\left(X^{*}\right)_{h}$ by taking $B^{\bullet}(R)^{*}=H^{\bullet}\left(S(R)^{*}\right)_{h}$, the Deligne mixed Hodge structure of the algebraic variety $S(R)^{*}$. We want to see that we have compatible morphisms of mixed Hodge complexes, $B^{\bullet}(R)^{*} \rightarrow B^{\bullet}(R)^{\mathrm{red}}$.

By (6.7), the connected components of $\bar{S}(R)^{\text {red }}$ are of the form

$$
{\overline{e^{\prime}(R)}}^{\mathrm{red}} \simeq\left(\overline{X_{\ell, R}}\right)^{\mathrm{red}} \times\left(\overline{M_{P}}\right)^{\mathrm{red}},
$$

with $P=\Pi(R)$. On the other hand, it is the algebro-geometric components of $S(R)^{*}$ that are of the form $\left(M_{P}\right)^{*}$, the Baily-Borel compactification of the lower-rank $M_{P}$. We note that (9.4.1) factors through the disjoint union

$$
\widetilde{S}(R)^{*}=: \bigsqcup\left(M_{P}\right)^{*},
$$

which is also an algebraic variety. Over $\left(M_{P}\right)^{*}$, the mapping $\bar{S}(R)^{\text {red }} \rightarrow \widetilde{S}(R)^{*}$ is given on connected components by the composition

$$
\left(\overline{X_{\ell, R}}\right)^{\text {red }} \times\left(\overline{M_{P}}\right)^{\text {red }} \rightarrow\left(\overline{M_{P}}\right)^{\text {red }} \rightarrow\left(M_{P}\right)^{*},
$$

where $\left(\overline{M_{P}}\right)^{\text {red }} \rightarrow\left(M_{P}\right)^{*}$ is the corresponding (quotient) mapping for $M_{P}$. Thus the mapping on cohomology induced by (9.4.3):

$$
H^{\bullet}\left(\left(M_{P}\right)^{*}\right) \rightarrow H^{\bullet}\left(\left(\overline{X_{\ell, R}}\right)^{\mathrm{red}}\right) \otimes H^{\bullet}\left(\left(\overline{M_{P}}\right)^{\mathrm{red}}\right)
$$

takes its values in $H^{0}\left(\left(\overline{X_{\ell, P}}\right)^{\text {red }}\right) \otimes H^{\bullet}\left(\left(\overline{M_{P}}\right)^{\text {red }}\right) \simeq H^{\bullet}\left(\left(\overline{M_{P}}\right)^{\text {red }}\right)$. We see that (9.4.4) is induced by

$$
H^{\bullet}\left(\left(M_{P}\right)^{*}\right) \rightarrow H^{\bullet}\left(\left(\overline{M_{P}}\right)^{\mathrm{red}}\right),
$$

which we are assuming, by induction, is a morphism of mixed Hodge structures, compatible with restrictions. This gives that

$$
H^{\bullet}\left(\widetilde{S}(R)^{*}\right) \rightarrow H^{\bullet}\left(\bar{S}(R)^{\mathrm{red}}\right)
$$

is a morphism of mixed Hodge structures. On the other hand, it follows directly from Deligne's mixed Hodge theory that

$$
H^{\bullet}\left(S(R)^{*}\right) \rightarrow H^{\bullet}\left(\widetilde{S}(R)^{*}\right)
$$

is a morphism of mixed Hodge structures. Combining these, we see that

$$
B^{\bullet}(R)^{*} \rightarrow B^{\bullet}(R)^{\text {red }}
$$


is a morphism of mixed Hodge complexes, compatible with restrictions, and we are done.

10. Towards mixed Hodge structures in the excentric Borel-Serre case. We would like to adapt the argument for $H^{\bullet}\left(\bar{X}^{\text {red }}\right)$ in (9.3) to the excentric Borel-Serre compactification $\bar{X}^{\text {exc }}$. However, we are no longer able to use $(2.10$,ii) to simplify the discussion, for the following reason. Equivariant cohomology is a composite functor:

$$
H_{\Gamma}^{\bullet}\left(K_{h}^{\bullet}\right)=H^{\bullet}\left(C^{\bullet}\left(\Gamma, K_{h}^{\bullet}\right)\right),
$$

and this does not generally coincide with $H^{\bullet}\left(\Gamma, H_{h}^{\bullet}\right)$. Indeed, the latter is the $E_{2}$-term of a spectral sequence abutting to (10.1), so it is of limited Hodge theoretic use even when the spectral sequence degenerates at $E_{2}$; there are non-trivial extensions in the category of mixed Hodge structures. What makes it work in the right-hand column of (9.3.2), which one can write as

$$
H_{\Gamma\left(G_{\ell, R}\right)}^{\bullet}\left(M_{P}^{\prime}, \mathbb{Q}\right) \simeq H^{\bullet}\left(\Gamma\left(G_{\ell, R}\right), H^{\bullet}\left(M_{P}, H^{\bullet}\left(\mathfrak{w}_{P}, \mathbb{Q}\right)\right)\right),
$$

is the theorem of Kostant from [Ko]. We leave it to the reader to pursue this.

In this Section we seek to adapt the discussion for $\bar{X}^{\text {red }}$ in $(9.3)$ to $\bar{X}^{\text {exc }}$. As suggested in $\S 6$, the original Borel-Serre construction [BS] applies to (connected) mixed Shimura varieties. ${ }^{10}$ The latter have excentric Borel-Serre compactifications whenever " $W_{-2}$ is trivial" (see [P:2.1(v)]), for example, $\mathcal{A}_{P}$. These are compact torus (Abelian) fibrations over a locally symmetric variety. To effect our induction, it is necessary to extend the construction of mixed Hodge structures to such mixed Shimura varieties. ${ }^{11}$ Specifically,

(10.3) Construction (anticipated mixed Hodge complexes for the excentric Borel-Serre). Let $X$ be a locally symmetric variety, or more generally, a mixed Shimura variety for which $\bar{X}^{\mathrm{exc}}$ is defined as a compactification of $X$. Take $A^{\bullet}$ and $C^{\bullet}(R)$ and $A^{\bullet} \rightarrow C^{\bullet}$ as in (9.3), though without taking cohomology, and put

$$
B^{\bullet}(R)=B^{\bullet}(R)^{\mathrm{exc}}=C^{\bullet}\left(\Gamma\left(G_{\ell, R}\right), C^{\bullet}\left(\left(\overline{\mathcal{A}_{P}}\right)^{\mathrm{exc}}\right)_{h}\right),
$$

where $C^{\bullet}\left(\left(\overline{\mathcal{A}_{P}}\right)^{\mathrm{exc}}\right)_{h}$ is the mixed Hodge complex for $H^{\bullet}\left(\left(\overline{\mathcal{A}_{P}}\right)^{\mathrm{exc}}\right)$ already constructed. Let $B^{\bullet}(R) \rightarrow C^{\bullet}(R)$ be the composite mapping

$$
\begin{aligned}
C^{\bullet}\left(\Gamma\left(G_{\ell, R}\right), C^{\bullet}\left(\left(\overline{\mathcal{A}_{P}}\right)^{\mathrm{exc}}\right)_{h}\right) \rightarrow C^{\bullet}\left(\Gamma\left(G_{\ell, R}\right), C^{\bullet}\left(\left(\overline{\mathcal{A}_{P}}\right)\right)_{h}\right) & \\
& \rightarrow C^{\bullet}\left(\Gamma\left(G_{\ell, R}\right), C^{\bullet}\left(\overline{M_{P}^{\prime}}\right)_{h}\right)
\end{aligned}
$$

Then the above data is in the category $\mathfrak{A}$ and moreover is expected to determine a mixed Hodge complex $C^{\bullet}\left(\bar{X}^{\mathrm{exc}}\right)_{h}$ for $H^{\bullet}\left(\bar{X}^{\mathrm{exc}}\right)$, such that the restriction $H^{\bullet}\left(\bar{X}^{\mathrm{exc}}\right) \rightarrow$ $H^{\bullet}\left(B^{\bullet}(R)^{\text {exc }}\right)$ to a closed boundary stratum and the natural mapping $H^{\bullet}\left(\bar{X}^{\mathrm{red}}\right) \rightarrow$ $H^{\bullet}\left(\bar{X}^{\mathrm{exc}}\right)$ are morphisms of mixed Hodge structures.

Explanation (incomplete). First, we note that the formula for $B^{\bullet}(R)^{\text {exc }}$ comes from (6.20). The outline for verifying (10.3) is largely the same as that of (9.3).

\footnotetext{
${ }^{10}$ We will make the following abuse of language. The standard usage, in the case of the mixed Shimura variety associated to $P$, involves complexifying $U_{P}$. However, we will decline to do that and use $U_{P}(\mathbb{R})$ instead of $U_{P}(\mathbb{C})$. This yields a space of the same homotopy type.

${ }^{11}$ Recall from $\S 6$ that there is no compactification " $\overline{\mathcal{A}_{P}}$ red" of $\mathcal{A}_{P}$ unless $V_{P}$ is trivial, so we do not have to extend (9.3).
} 
There is only one diagram that requires serious checking, namely the one obtained by inserting a central column in item ii) from the proof of $(9.3)$ and forgetting $(2.10$, ii):

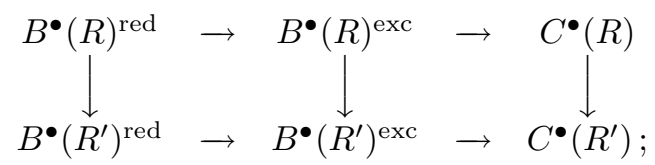

it is to be a commutative diagram of mixed Hodge complexes. Explicitly, this is

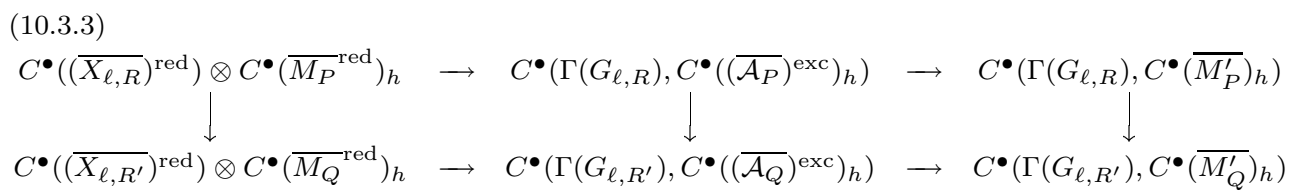

We may replace $C^{\bullet}\left(\left(\overline{X_{\ell, R}}\right)^{\text {red }}\right) \otimes C^{\bullet}\left({\overline{M_{P}}}^{\text {red }}\right)_{h}$ by $C^{\bullet}\left(\Gamma\left(G_{\ell, R}\right), C^{\bullet}\left({\overline{M_{P}}}^{\text {red }}\right)_{h}\right)$, etc., for the former maps into this anyway. This gives, replacing (10.3.3),

$(10.3 .4)$

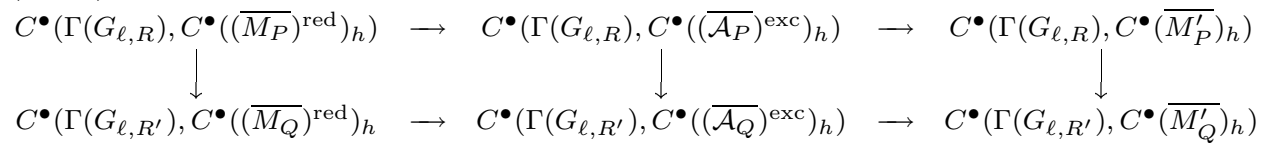

As before, the case $Q=P$ is very easy, the rows being morphisms by induction, and the columns are given by restriction to a subgroup. When $\Pi\left(R^{\prime}\right)=Q \prec P$, we rewrite (10.3.4) relative to $P$, where we understand that we are taking $\Gamma\left(G_{\ell, R}\right)$-cochains throughout:

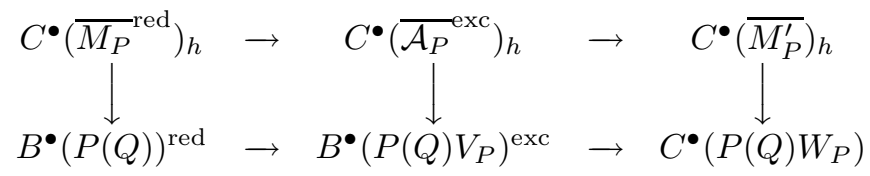

To obtain the bottom row here (in the unfiltered sense first) from that of (10.3.4), one makes use of (8.12) and (6.A.1); the most interesting case is:

$$
\begin{aligned}
& C^{\bullet}\left(\Gamma\left(G_{\ell, R^{\prime}}\right), C^{\bullet}\left(\left(\overline{\mathcal{A}_{Q}}\right)^{\mathrm{exc}}\right)\right) \\
& \begin{aligned}
& \approx C^{\bullet}\left(\Gamma\left(G_{\ell, R}\right), C^{\bullet}\left(\Gamma\left(W_{Q(P)}\right) \Gamma\left(G_{\ell, P(Q)}\right), C^{\bullet}\left(\Gamma\left(V_{Q}\right), C^{\bullet}\left(\left(\overline{M_{Q}}\right)^{\mathrm{exc}}\right)\right)\right)\right. \\
& \approx C^{\bullet}\left(\Gamma\left(G_{\ell, R}\right), C^{\bullet}\left(\Gamma\left(V_{P}\right) \Gamma\left(V_{P(Q)}\right), C^{\bullet}\left(\Gamma\left(G_{\ell, P(Q)}\right), C^{\bullet}\left({\overline{M_{Q}}}^{\mathrm{exc}}\right)\right)\right)\right) \\
&\left.\approx C^{\bullet}\left(\Gamma\left(G_{\ell, R}\right), C^{\bullet}\left({\overline{e^{\prime}\left(P(Q) V_{P}\right)}}^{\mathrm{exc}}\right)\right)\right) .
\end{aligned}
\end{aligned}
$$

The rows of (10.3.5) are again morphisms of mixed Hodge complexes by induction, and the columns are given by restriction to a boundary face, which is also a morphism by induction.

What we need in order to continue is knowing that the induced morphisms of mixed Hodge structure on cohomology are the same as those of (10.3.4); that is, that the change of base between $P$ and $Q$ in (10.3.6) is Hodge theoretical (recall (8.17)). I suspect that we lack sufficient methods to prove this now. (See $\S 12$ below for some ideas.) 
Finally, since we have used for each $Q$ a different mixed Hodge complex for each $P \succeq Q$, we would appeal to (2.12) to finish the construction.

We assert a consequence of the construction in (10.3), which is conditional upon completing the argument:

(10.4) Assertion. Let $X_{1}$ and $X_{2}$ be mixed Shimura varieties of the type in (10.3), and suppose there is a morphism of mixed Shimura varieties $X_{1} \rightarrow X_{2}$. Then the induced morphism $H^{\bullet}\left(\bar{X}_{2}^{\text {exc }}\right) \rightarrow H^{\bullet}\left(\bar{X}_{1}^{\text {exc }}\right)$ is a morphism of mixed Hodge structures.

Proof (assuming (10.3)). It goes by induction. Let $\mathcal{A}_{P, 1}$ and $\mathcal{A}_{P, 2}$ be the respective mixed Shimura varieties in the description of $\bar{X}_{1}^{\mathrm{exc}}$ and $\bar{X}_{2}^{\mathrm{exc}} ;$ these are the ones for which

$$
\begin{aligned}
B^{\bullet}\left(X_{2} ; R\right)^{\mathrm{exc}} & =C^{\bullet}\left(\Gamma\left(G_{\ell, R}\right), C^{\bullet}\left(\left(\overline{\mathcal{A}_{P, 2}}\right)^{\mathrm{exc}}\right)_{h}\right) \\
\downarrow & B^{\bullet}\left(X_{1} ; R\right)^{\mathrm{exc}}=C^{\bullet}\left(\Gamma\left(G_{\ell, R}\right), C^{\bullet}\left(\left(\frac{\downarrow}{\left(\mathcal{A}_{P, 1}\right.}\right)^{\mathrm{exc}}\right)_{h}\right)
\end{aligned}
$$

Because $C^{\bullet}\left(\left(\overline{\mathcal{A}_{P, 2}}\right)^{\text {exc }}\right)_{h} \rightarrow C^{\bullet}\left(\left(\overline{\mathcal{A}_{P, 1}}\right)^{\text {exc }}\right)_{h}$ is a morphism of mixed Hodge complexes for all $P$, so is $B^{\bullet}\left(X_{2}\right)^{\text {exc }} \rightarrow B^{\bullet}\left(X_{1}\right)^{\text {exc }}$.

(10.5) Remark. Note that we did not use for (10.4) that the change of base was Hodge-theoretic beyond what was needed for (10.3).

Accepting (10.3) again, we obtain immediately from (9.4) that:

(10.6) Corollary. Assume that the construction in (10.3) is completed. Then the mapping $H^{\bullet}\left(X^{*}\right) \rightarrow H^{\bullet}\left(\bar{X}^{\mathrm{exc}}\right)$ is a morphism of mixed Hodge structures.

11. Towards mixed Hodge structures in the excentric toroidal case. In this section, the construction from $\S 10$ will get mimicked for the excentric toroidal compactification. Since $X^{\text {tor }}$ is (with our convention) a projective manifold, its cohomology has classical Hodge theory. It is for the excentric toroidal compactification $X^{\text {tor,exc }}$, again a space that usually has odd-dimensional boundary strata, that we (conditionally) produce something new.

The construction of toroidal compactifications extend to mixed Shimura varieties, as is carried out in $[\mathrm{P}]$. Their excentric toroidal compactifications are defined when $W_{-2}$ is trivial-precisely when $\bar{X}^{\text {exc }}$ is. Making parallel use of that as part of the induction, we get the toroidal analogue of (10.3):

(11.1) Construction (anticipated mixed Hodge complexes in the excentric toroidal case). Let $X$ be a locally symmetric variety, or more generally, a mixed Shimura variety for which $X^{\text {tor,exc }}$ is defined. Take $A^{\bullet}$ and $C^{\bullet}(R)$ as in (9.3), and put

$$
B^{\bullet}(R)=B^{\bullet}(R)^{\mathrm{tor}, \mathrm{exc}}:=C^{\bullet}\left(\Gamma\left(G_{\ell, R}\right), C^{\bullet}\left(\left(\mathcal{A}_{P}\right)^{\mathrm{tor}, \mathrm{exc}}\right)_{h}\right),
$$

where $C^{\bullet}\left(\left(\mathcal{A}_{P}\right)^{\text {tor,exc }}\right)_{h}$ is the mixed Hodge complex for $H^{\bullet}\left(\left(\overline{\mathcal{A}_{P}}\right)^{\text {tor,exc }}\right)$ already constructed. Let $B^{\bullet}(R) \rightarrow C^{\bullet}(R)$ be the composite mapping

$$
\begin{aligned}
C^{\bullet}\left(\Gamma\left(G_{\ell, R}\right), C^{\bullet}\left(\left(\mathcal{A}_{P}\right)^{\text {tor }, \text { exc }}\right)_{h}\right) \rightarrow C^{\bullet}\left(\Gamma\left(G_{\ell, R}\right), C^{\bullet}\right. & \left.\left(\left(\mathcal{A}_{P}\right)^{\text {tor }}\right)_{h}\right) \\
& \rightarrow C^{\bullet}\left(\Gamma\left(G_{\ell, R}\right), C^{\bullet}\left(\overline{M_{P}^{\prime}}\right)_{h}\right)
\end{aligned}
$$


Then the above data is in the category $\mathfrak{A}$ and moreover is expected to determine a mixed Hodge complex $C^{\bullet}\left(X^{\text {tor,exc }}\right)_{h}$ for $H^{\bullet}\left(X^{\text {tor,exc }}\right)$, such that the restriction $H^{\bullet}\left(X^{\text {tor,exc }}\right) \rightarrow H^{\bullet}\left(B^{\bullet}(R)^{\text {tor,exc }}\right)$ to a closed boundary stratum and the natural mapping $H^{\bullet}\left(X^{\text {tor,exc }}\right) \rightarrow H^{\bullet}\left(X^{\text {tor }}\right)$ are morphisms of mixed Hodge structures. Here, $H^{\bullet}\left(X^{\text {tor }}\right)$ is understood to have the classical pure Hodge structures for the smooth projective variety $X^{\text {tor }}$.

Explanation (incomplete). We follow an argument analogous to the one for (10.3). Here, we must first reconstitute the mixed Hodge structure on $H^{\bullet}\left(X^{\text {tor }}\right)$ along the lines of our construction; this is done by taking $B^{\bullet}(R)^{\text {tor }}$ to be $C^{\bullet}(Z(R))_{h}$. The mapping $B^{\bullet}(R)^{\text {tor,exc }} \rightarrow B^{\bullet}(R)^{\text {tor }}$ is defined as the canonical composition

$$
\left.C^{\bullet}\left(\Gamma\left(G_{\ell, R}\right), C^{\bullet}\left(\mathcal{A}_{P}\right)^{\text {tor }, \text { exc }}\right)\right) \rightarrow C^{\bullet}\left(\Gamma\left(G_{\ell, R}\right), C^{\bullet}(\widetilde{Z}(R))\right) \rightarrow C^{\bullet}(Z(R)) .
$$

In the present instance, the essential diagram to check (cf. (10.3.2)) is

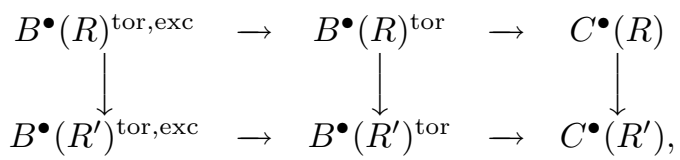

Explicitly, this is

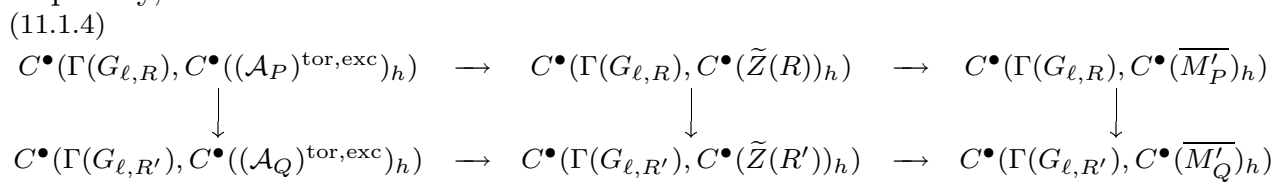

The case $Q=P$ is again easy, with the rows assumed to be morphisms of mixed Hodge structure by the proposed induction, and the columns given by restriction to a subgroup. In the case where $\Pi\left(R^{\prime}\right)=Q \prec P$, we proceed a little differently from (10.3.4). Concerning the interesting left-hand square, we note that the diagram (8.14) factors, by the toroidal construction itself, through

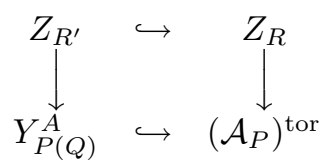

where $Y_{P(Q)}^{A}$ is the boundary divisor in $\left(\mathcal{A}_{P}\right)^{\text {tor }}$ corresponding to $P(Q) V_{P} \subset G_{h, P} V_{P}$; $Y_{P(Q)}^{A}$ projects onto $Y_{P(Q)}$ in $\left(M_{P}\right)^{\text {tor }}$. The excentric quotient of (11.1.5) can be taken. This yields the diagram:

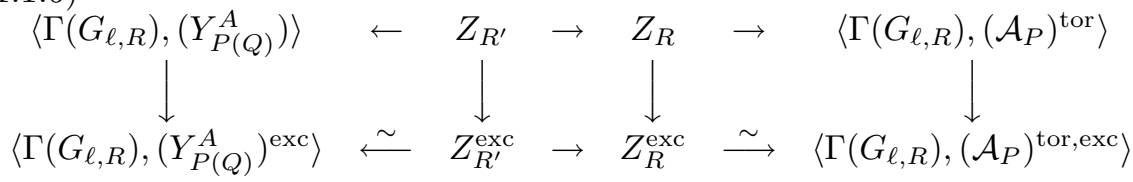

Furthermore, we have an analogue of (11.1.6) coming from (7.3):

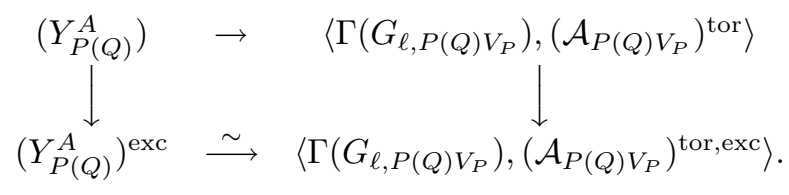


When we take the cochain complexes for the spaces in (11.1.6) and (11.1.7), we would have (by induction) morphisms of mixed Hodge complexes. As was the case for (10.3), we would need that these morphisms of mixed Hodge structure are the same as those in (11.1.4), i.e., that the change of base between $P$ and $Q$ here is Hodge theoretical (8.17). Without that, the induction is, once again, stalled.

The next natural step is to give the analogue of (10.4), and that is conditional upon completing the argument for (11.1):

(11.2) Assertion. Let $X_{1}$ and $X_{2}$ be mixed Shimura varieties of the type in (10.3), and suppose there is a morphism of mixed Shimura varieties $X_{1} \rightarrow X_{2}$. Then the induced morphism $H^{\bullet}\left(X_{2}^{\text {tor,exc }}\right) \rightarrow H^{\bullet}\left(X_{1}^{\text {tor,exc }}\right)$ should be a morphism of mixed Hodge structures.

Proof (assuming the completion of the argument for (11.1)). We argue by induction. Let $\mathcal{A}_{P, 1}$ and $\mathcal{A}_{P, 2}$ be the respective mixed Shimura varieties in the description of $X_{1}^{\text {exc }}$ and $X_{2}^{\text {tor,exc }}$; these are the ones for which

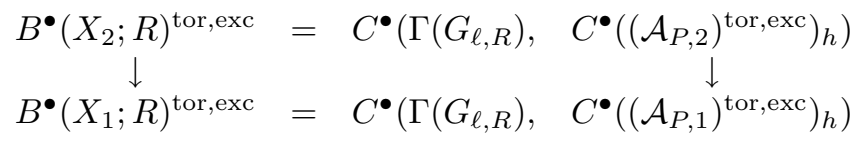

Because $\left.\left.C^{\bullet}\left(\left(\overline{\mathcal{A}_{P, 2}}\right)^{\text {exc }}\right)_{h}\right) \rightarrow C^{\bullet}\left(\left(\overline{\mathcal{A}_{P, 1}}\right)^{\text {exc }}\right)_{h}\right)$ is a morphism of mixed Hodge complexes for all $P$, so is $B^{\bullet}\left(X_{2}\right)^{\text {exc }} \rightarrow B^{\bullet}\left(X_{1}\right)^{\text {exc }}$.

The analogue of (10.6) should also hold in the toroidal case. To make the analogy clear, we note that the proof of (9.4) comes down to the assertion that the mapping ${\overline{M_{P}}}^{\text {red }} \rightarrow M_{P}^{*}$ induces a morphism of mixed Hodge structure on cohomology, and (11.2) is about the corresponding statement for $\overline{M_{P}}{ }^{\mathrm{exc}} \rightarrow M_{P}^{*}$. Therefore, by composition with ${\overline{\mathcal{A}_{P}}}^{\text {exc }} \rightarrow{\overline{M_{P}}}^{\text {exc }}$, the mapping ${\overline{\mathcal{A}_{P}}}^{\text {exc }} \rightarrow M_{P}^{*}$ would also induce a morphism of mixed Hodge structure on cohomology.

(11.3) Assertion. The mapping $H^{\bullet}\left(X^{*}\right) \rightarrow H^{\bullet}\left(X^{\text {tor,exc }}\right)$ should be a morphism of mixed Hodge structure.

Proof (assuming the completion of the argument for (11.1)). We must consider the mappings

$$
\left\langle\Gamma\left(G_{\ell, R}\right),\left(\mathcal{A}_{P}\right)^{\text {tor,exc }}\right\rangle \rightarrow M_{P}^{*}
$$

for $P$ maximal. This leads us to the more fundamental mapping $\left(\mathcal{A}_{P}\right)^{\text {tor,exc }} \rightarrow M_{P}^{*}$, which factors through $\left(M_{P}\right)^{\mathrm{tor}, \mathrm{exc}}$. The mapping $\left(\mathcal{A}_{P}\right)^{\mathrm{tor}, \mathrm{exc}} \rightarrow\left(M_{P}\right)^{\mathrm{tor}, \mathrm{exc}}$ induces a morphism of mixed Hodge structures by induction, as does $\left(M_{P}\right)^{\text {tor,exc }} \rightarrow M_{P}^{*}$.

Next, there are continuous mappings $k: X^{\text {tor,exc }} \rightarrow \bar{X}^{\text {exc }}$, defined in [Z4:(3.4)], partly along the lines of $[\mathrm{GT}]$. We showed that the two spaces are, thereby, homotopy equivalent. It should be possible to reprove this result by a geometric recursion, using (6.19), (7.3) and the correspondence of deleted neighborhoods of the boundary nerves (from [HZ2:§2]; see also (6.14)). We expect that we will then be able to prove:

(11.4) Assertion. The isomorphism $k^{*}: H^{\bullet}\left(\bar{X}^{\mathrm{exc}}\right) \simeq H^{\bullet}\left(X^{\mathrm{tor}, \mathrm{exc}}\right)$ is a morphism of mixed Hodge structures. 
12. Additional features and hopes. In what follows, Hodge-theoretic statements about the excentric compactifications are contingent upon completing the arguments for the constructions in $\S 10$ and $\S 11$.

We will show first that the range of weights occurring in the mixed Hodge structures from $\S 9-\S 11$ will be the same as those of a complete algebraic variety. It is convenient to give first a general feature of our construction. For compactifications $Y$ of $X$, we are considering mixed Hodge complexes of the form

$$
K^{\bullet}(Y)=\operatorname{Cone}\left\{A^{\bullet}(X) \oplus B^{\bullet}(\partial Y) \rightarrow C_{\mathrm{dn}}^{\bullet}(\partial Y)\right\},
$$

with the understanding that the complexes $C^{\bullet}(X)$ and $C_{\mathrm{dn}}^{\bullet}(\partial Y)$ are taken independent of $Y$. We then have:

(12.2) Lemma. Let $Y_{1} \rightarrow Y_{2}$ be a morphism of compactifications of $X$. Let $B^{\bullet}\left(\partial Y_{1}\right)$ and $B^{\bullet}\left(\partial Y_{2}\right)$ be mixed Hodge complexes for their respective boundaries with a morphism of mixed Hodge complexes $B^{\bullet}\left(\partial Y_{2}\right) \rightarrow B^{\bullet}\left(\partial Y_{1}\right)$. Finally, let $K^{\bullet}\left(Y_{1}\right)$ and $K^{\bullet}\left(Y_{2}\right)$ be given by the mixed cone (12.1). Then:

i) this induces a morphism of mixed Hodge complexes $K^{\bullet}\left(Y_{2}\right) \rightarrow K^{\bullet}\left(Y_{1}\right)$;

ii) the two mixed cones

$$
\text { Cone }\left\{K^{\bullet}\left(Y_{2}\right) \rightarrow K^{\bullet}\left(Y_{1}\right)\right\} \quad \text { and } \quad \text { Cone }\left\{B^{\bullet}\left(\partial Y_{2}\right) \rightarrow B^{\bullet}\left(\partial Y_{1}\right)\right\}
$$

are quasi-isomorphic, and their filtrations induce the same mixed Hodge structure on cohomology.

Proof. This is quite straightforward. The existence of a bifiltered mapping $K^{\bullet}\left(Y_{2}\right)$ $\rightarrow K^{\bullet}\left(Y_{1}\right)$ is automatic, giving i). Next, $K^{\bullet}\left(Y_{1}\right)$ and $K^{\bullet}\left(Y_{2}\right)$ differ only in the term $B^{\bullet}(\partial Y)$. Up to quasi-isomorphism, the other terms "cancel," leaving the complex Cone $\left\{B^{\bullet}\left(\partial Y_{2}\right) \rightarrow B^{\bullet}\left(\partial Y_{1}\right)\right\}$. Thus, we have verified ii).

(12.3) Proposition. The weights occurring in $H^{i}\left(\bar{X}^{\mathrm{red}}\right)$ are $\leq i$, i.e., $C^{\bullet}\left(\bar{X}^{\mathrm{red}}\right)_{h}$ is of complete type. The same holds for $H^{i}\left(\bar{X}^{\mathrm{exc}}\right)$ and $H^{i}\left(X^{\mathrm{tor}, \mathrm{exc}}\right)$ if the arguments for (10.3) and (11.1) are completed.

Proof. We prove this by induction (of course). Since we are considering spaces with a simplicial boundary structure, they all follow a single line. We apply $(12.2$, ii $)$ to the cases $X^{\text {tor }} \rightarrow X^{\text {tor,exc }}$ and $\bar{X}^{\text {exc }} \rightarrow \bar{X}^{\text {red }}$, using (11.4) to connect the two. Then, the conclusion about weights passes from $H^{i}\left(X^{\text {tor }}\right)$ to $H^{i}\left(X^{\text {tor,exc }}\right)$ by $(2.14)$, and likewise from $H^{i}\left(\bar{X}^{\mathrm{exc}}\right)$ to $H^{i}\left(\bar{X}^{\mathrm{red}}\right)$.

It is natural to ask whether the analogue of [De:II,(3.2.17)] is true:

$$
\begin{aligned}
& \text { (12.4) Question. Is } W_{i} H^{i}(X)=i m\left\{H^{i}(\widehat{X}) \rightarrow H^{i}(X)\right\} \text { for } \widehat{X} \text { equaling } \bar{X}^{\text {red }} \text {, } \\
& \bar{X}^{\mathrm{exc}} \text {, and } X^{\text {tor,exc? }}
\end{aligned}
$$

Note, however, that the assertion is clearly false for $\widehat{X}=\bar{X}$.

Next, if $E$ is a homogeneous vector bundle on $X$, it has canonical extensions $\bar{E}^{\text {red }}$ to $\bar{X}^{\text {red }}$ (and then $\bar{E}^{\text {exc }}$ to $\bar{X}^{\text {exc }}$ ), and also $E^{\text {tor,exc }}$ to $X^{\text {tor,exc }}$. It should not be hard to show that the Chern classes of $\bar{E}^{\text {red }}$ have the usual property: $c_{p}\left(\bar{E}^{\text {red }}\right)$ lies in $F^{p} W_{2 p} H^{2 p}\left(\bar{X}^{\text {red }}\right)$ and $c_{p}\left(E^{\text {tor }, \text { exc }}\right)$ lies in $F^{p} W_{2 p} H^{2 p}\left(X^{\text {tor }, \text { exc }}\right)$. 
Last along these lines, we expect it is not hard to see that all of the mixed Hodge structures we have constructed (to the extent that they have been) have natural polarizations (in the usual graded sense).

The notion we called Hodge-theoretic change of base (8.17) was our only obstruction, though a serious one, to defining mixed Hodge structures on the cohomology of the excentric Borel-Serre compactification and the excentric toroidal compactification. A plausible idea would be to define a notion of mixed Hodge modules on such spaces (or more generally). The decomposition theorem from algebraic geometry plays a central role in the construction in [Sa1]. I used to daydream about generalizing the decomposition theorem to a larger class of spaces than complex Kähler varieties. Following the lines suggested in this paragraph might be a good application of, hence motivation for, an extended notion of mixed Hodge modules.

\section{REFERENCES}

[AMrT] Ash, A., Mumford, D., Rapoport, M., Tai, Y.-S., Smooth Compactification of Locally Symmetric Varieties, Math. Sci. Press, Brookline, MA, 1975.

[BB] Baily, W., Borel, A., Compactification of arithmetic quotients of bounded symmetric domains, Ann. of Math., 84 (1966), pp. 442-528.

[Be] Beilinson, A., Notes on absolute Hodge cohomology, In: Applications of Algebraic KTheory to Algebraic Geometry and Number Theory, Part I. Contemporary Mathematics 55, AMS, 1986.

[Bo] Borel, A., Introduction aux Groupes Arithmétiques, Hermann, Paris, 1969.

[BS] Borel, A., Serre, J.-P., Corners and arithmetic groups, Comm. Math. Helv., 4 (1973), pp. $436-491$.

[BT] Borel, A., Tits, J., Groupes réductifs, Publ. Math. IHES, 27 (1965), pp. 55-151.

[BW] Burgos Gil, J., Wildeshaus, J., Hodge modules on Shimura varieties and their higher direct images in the Baily-Borel compactification, (to appear).

[De] Deligne, P., Théorie de Hodge, II III, Publ. Math. IHES, 40 (1971), pp. 5-57; 44 (1974), pp. $5-77$.

[E] El Zein, F., Mixed Hodge structures, Trans. AMS, 275 (1983), pp. 71-106.

[GHM] Goresky, M., Harder, G., MacPherson, R., Weighted cohomology, Invent. Math., 116 (1994), pp. 139-213.

[GT] Goresky, M., TAI, Y.-S., Toroidal and reductive Borel-Serre compactifications of locally symmetric spaces, Amer. J. Math., 121 (1999), pp. 1095-1151.

[HZ1] Harris, M., Zucker, S., Boundary cohomology of Shimura varieties, I: Coherent cohomology on toroidal compactifications, Ann. Sci. ENS, 27 (1994), pp. 249-344.

[HZ2] Harris, M., Zucker, S., Boundary cohomology of Shimura varieties, II: Hodge theory at the boundary, Invent. Math., 116 (1994), pp. 243-307.

[HZ3] Harris, M., Zucker, S., Boundary cohomology of Shimura varieties, III: Coherent cohomology on higher-rank boundary strata and applications to Hodge theory, Mem. Soc. Math. France, 85 (2001), pp. 116.

[K] Kostant, B., Lie algebra cohomology and the generalized Borel-Weil theorem, Ann. of Math., 74 (1961), pp. 329-287.

[NA] Navarro Aznar, V., Sur la théorie de Hodge-Deligne, Invent. Math., 90 (1987), pp. 11-76.

[P] Pink, R., Arithmetical compactifications of mixed Shimura varieties, Bonn Math. Schrift., Nr. 209 (1990).

[Sa1] Saito, Mo., Mixed Hodge modules, Publ. RIMS Kyoto Univ., 26 (1990), pp. 221-333.

[Sa2] SAito, Mo., Mixed Hodge modules and admissible variations, C.R.A.S. Paris, 309 (1989), pp. 351-356.

[S] SAPER, L., L-modules and micro-support, 2002.

[Z1] Zucker, S., L $L_{2}$-cohomology of warped products and arithmetic groups, Invent. Math., 70 
(1982), pp. 169-218.

[Z2] Zucker, S., Satake compactifications, Commentarii Math. Helvetica, 58 (1983), pp. 312343 (Erratum on p. 337 of $L^{p}$-cohomology and Satake compactifications, J. Noguchi, T. Ohsawa (eds.), Prospects in Complex Geometry: Proceedings, Katata/ Kyoto 1989, Springer LNM, 1468 (1991), pp. 317-339).

[Z3] Zucker, S., On the reductive Borel-Serre compactification: $L^{p}$-cohomology of arithmetic groups (for large p), Amer. J. Math., 123 (2001), pp. 951-984.

[Z4] ZuCKER, S., On the reductive Borel-Serre compactification II: Excentric quotients and least common modifications, (to appear). 
S. ZUCKER 